\title{
A NEW CONSTRUCTION OF NONCROSSED PRODUCT ALGEBRAS
}

BY

\author{
BILL JACOB ${ }^{1}$ AND ADRIAN R. WADSWORTH ${ }^{2}$
}

\begin{abstract}
New examples of noncrossed product division algebras are obtained, using methods different from all previous noncrossed product constructions. The examples are division algebras over intersections of $p$-Henselian valued fields, and they have Schur index $p^{m}$ and exponent $p^{n}$ for any prime number $p$ and any integers $m \geqslant n \geqslant 2(n \geqslant 3$ if $p=2)$. The basic tools used in the construction are valuation theory and Galois cohomology; no generic methods are applied and there is no p.i. theory. Along the way, local-global principles are proved for central simple algebras over intersections of $p$-Henselian valued fields.
\end{abstract}

The first examples of central simple division algebras which are not crossed products were obtained by Amitsur in [Am] in 1972. Amitsur thereby settled a question that had been one of the outstanding open problems in the theory of algebras for at least thirty years. His examples were the generic division algebras $U D(\mathbf{Q}, n)$ of index $n$ over $\mathbf{Q}$ (the rational numbers) for any natural number $n$ such that $p^{2} \mid n, p$ an odd prime, or $8 \mid n$. All subsequent constructions of noncrossed products in [SS, $\mathbf{S a}_{1}-\mathbf{S a}_{3}, \mathbf{R i}, \mathbf{R o}$, and $\mathbf{T i}$ ] have heretofore been based on Amitsur's specialization argument, and they are all generic division algebras or extensions of generic division algebras. The centers of these noncrossed product algebras are not known, nor are the Brauer groups, nor the absolute Galois groups of the centers.

We present here a new method of constructing noncrossed product algebras. In our approach, the noncrossed product is realized as the underlying division algebra $D$ of a tensor product of suitably chosen cyclic algebras over a field $F=L_{1} \cap L_{2}$, where each $L_{i}$ is a $p$ th root Henselian valued field. We prove local global principles relating the splitting fields of $D$ to those of $D \otimes_{F} L_{i}, i=1,2$. It is shown that the $p$-part of the Brauer group of $F$ is completely determined by that of $L_{1}$ and $L_{2}$. Computations for central simple $F$-algebras thus become very tractable. For example, we not only show that $D$ is not a crossed product, but also calculate exactly how large $r$ must be so that the matrix ring $M_{r}(D)$ is a crossed product, and how large $s$ must be so that $M_{s}(D)$ is a tensor product of cyclic algebras. Indeed, the structure of the Brauer group of $F$ is so nice that we were somewhat surprised that noncrossed products could possibly exist over $F$.

Received by the editors March 19, 1985.

1980 Mathematics Subject Classification. Primary 16A39; Secondary 12J10, 12G05.

${ }^{1}$ This collaboration resulted from the first author's visit to U.C.S.D. during 1984-85. He would like to thank the U.C.S.D. Mathematics Department for its hospitality. He was also supported in part by the N.S.F.

${ }^{2}$ Supported in part by the N.S.F.

(C)1986 American Mathematical Society $0002-9947 / 86 \$ 1.00+\$ .25$ per page 
The paper is organized as follows: In $\S 1$ we define terminology and describe the $p$-Galois cohomology and the $p$ th root Henselian valuations that will be used throughout the paper. We develop in $\$ \S 2$ and 3 the "local" theory of valued division algebras and the $p$-Brauer group of fields with $p$ th root Henselian valuations. The bridge between the "local" theory for $p$ th root Henselian valued fields $L_{i}$ and the "global" theory for $L_{1} \cap L_{2}$ is provided in $\S 4$ : We prove that (under suitable hypotheses) the $p$-part of the absolute Galois group of $L_{1} \cap L_{2}$ is the free product (in the category of pro-p-groups) of the $p$-parts of the absolute Galois groups of $L_{1}$ and $L_{2}$ (Theorem 4.3). Finally in $\$ \S 5$ and 6 we give the noncrossed product examples. At the end of $\$ 5$ we indicate how the same methods yield examples of indecomposable algebras with index exceeding the exponent.

A number of results given here can be proved either by valuation theory or by cohomological methods. Both perspectives are worthwhile, and we will try to steer a middle course to give a good sampling of each approach.

\section{Preliminaries from the theory of algebras, Galois cohomology and valuation}

theory. All algebras considered in this paper will be finite dimensional over some field $F$. If $A$ is a central simple $F$-algebra, $[A]$ denotes the class of $A$ in the Brauer group $\operatorname{Br}(F)$ of $F$. We write $\exp (A)$ for the exponent of $A$, which is the order of $[A]$ in $\operatorname{Br}(F)$. By Wedderburn's theorem $A \cong M_{n}(D)$, i.e., $n \times n$ matrices over some $F$-central division algebra $D$. The integer $\sqrt{\operatorname{dim}_{F} D}$ is the (Schur) index of $A$, denoted index $(A)$. We will need the fact (cf. [R, Theorems 29.22, 29.24]) that $\exp (A) \mid \operatorname{index}(A)$ and $\exp (A)$ and $\operatorname{index}(A)$ have the same prime factors. It is standard that every maximal subfield $K$ of $D$ splits $A$ and $[K: F]=\operatorname{index}(A)$. More generally, we recall from [R, pp. 238-240, Theorem 28.5, Corollary 28.10]:

Let $A \cong M_{n}(D)$ be a central simple $F$-algebra ( $D$ the associated division algebra). If $L \supseteq F$ is a field with $[L: F]<\infty$ and $L$ splits $A$, then $[L: F]=s \cdot \operatorname{index}(A)$ for some integer $s$, and $L$ is isomorphic to a (maximal) subfield of $M_{s}(D)$. Conversely, if $K \supseteq F$ is any subfield of $M_{s}(D)$ and $[K: F]=s \cdot \operatorname{index}(A)$, then $K$ splits $A$.

Recall that a central simple $F$-algebra $A$ is a crossed product just when $A$ has a (maximal) subfield $M$ Galois over $F$, with $[M: F]^{2}=\operatorname{dim}_{F} A$. For such an algebra, the multiplication table on a base is completely determined by the multiplication in $M$, the Galois group $\mathscr{G}(M / F)$ and a 2-cocycle of $\mathscr{G}(M / F)$. It is through crossed products that one obtains the cohomological interpretation of the Brauer group: $\operatorname{Br}(F) \cong H^{2}\left(\mathscr{G}(\tilde{F} / F), \tilde{F}^{*}\right)$, where $\tilde{F}$ is a separable closure of $F$ (cf. [CF, pp. 125-126; R, p. 246, Theorem 29.12; or $\mathbf{S e}_{2}$, Chapter X, §§4-5]). Our strategy for constructing central simple algebras which are not crossed products is to produce an algebra $A$ with $\operatorname{dim}_{F} A=d^{2}$ so that $A$ has a splitting field of degree $t$ over $F, t \mid d$, but $A$ has no splitting field Galois over $F$ with degree dividing $t$. Then (1.1) shows that $A \cong M_{d / t}\left(A^{\prime}\right)$ for some central simple $F$-algebra $A^{\prime}$, but $A^{\prime}$ cannot be a crossed product, nor a matrix algebra over a crossed product.

The algebras $A$ in our example will be built from cyclic algebras, for which we will use the following notation: If $K$ is a Galois extension field of $F$ with $\mathscr{G}(K / F)$ cyclic 
of order $n$ with generator $\sigma$, and if $b \in F^{*}=F-\{0\}$, then $A(K / F, \sigma, b)$ denotes the ring generated over $K$ by an element $x$ subject to the relations $x k=\sigma(k) x$ for all $k \in K$, and $x^{n}=b$. Recall that $A(K / F, \sigma, b)$ is a central simple $F$-algebra of dimension $n^{2}$ over $F$ in which $K$ is a maximal subfield. A very nice account of cyclic algebras is given in $[\mathbf{R}, \S 30]$.

Suppose $F$ contains a primitive $n$th root of unity $\omega$. We write $A_{\omega}(a, b ; F)$ for the "symbol" determined by $a$ and $b$, i.e., the central simple $n^{2}$-dimensional $F$-algebra with generators $i, j$ and relations $i^{n}=a, j^{n}=b, i j=\omega j i$. Of course, Kummer theory shows that with $\omega \in F$ every cyclic $F$-algebra of dimension $n^{2}$ is a symbol.

Fix a prime number $p \neq \operatorname{char} F$. Let ${ }_{p^{n}} \operatorname{Br}(F)$ denote the subgroup of $\operatorname{Br}(F)$ consisting of those $[A]$ with $\exp (A) \mid p^{n}$, and let $\operatorname{Br}_{p}(F)=\bigcup_{n=1}^{\infty} p^{n} \operatorname{Br}(F)$, the $p$-primary component of $\operatorname{Br}(F)$. Our noncrossed product examples will all have exponent (hence index) a $p$-power. One reason for this is that key cohomological results in $\$ 4$ hold for pro-p-groups, but are not known for arbitrary profinite groups. It is easy to work from our examples to construct noncrossed products of composite exponent; we will not do so, preferring to focus attention on the more basic ideas involved in the construction.

For any profinite group $G$ and discrete $G$-module $M, H^{i}(G, M)$ denotes the $i$ th continuous cohomology group of $G$ with coefficients in $M$ (as described e.g., in [CF, Chapter V; Sh, Chapter II; $\mathbf{S e}_{\mathbf{1}}$, Chapter I; T, §2]). In particular, if $N$ is any closed subgroup of $G, \operatorname{res}_{G \rightarrow N}: H^{i}(G, M) \rightarrow H^{i}(N, M)$ denotes the restriction map; if $N$ is normal in $G$, then $\inf _{G / N \rightarrow G}: H^{i}\left(G / N, M^{N}\right) \rightarrow H^{i}(G, M)$ is the inflation map.

Given a field $F$ with char $F \neq p$, let $\mu_{p^{n}}$ denote the group of all $p^{n}$ th roots of unity in $\tilde{F}$, a separable closure of $F$. Then $\mu_{p^{n}}$ is a discrete module for the profinite group $G(F):=\mathscr{G}(\tilde{F} / F)$, and we recall the standard isomorphisms

$$
H^{1}\left(G(F), \mu_{p^{n}}\right) \cong F^{*} / F^{* p^{n}} \text { and } H^{2}\left(G(F), \mu_{p^{n}}\right) \cong_{p^{n}} \operatorname{Br}(F),
$$

which are derived the same way as (1.7) below, but with $\tilde{F}$ replacing $\tilde{F}_{p}$. For $a \in F^{*}$ we write $(a)\left(\right.$ or $\left.(a)_{F}\right)$ for the image of $a F^{* p^{n}}$ in $H^{1}\left(G(F), \mu_{p^{n}}\right)$.

Suppose now that $\mu_{p^{n}} \subseteq F$. Then we have the $G(F)$-module isomorphism $\mu_{p^{n}} \cong$ $\mathbf{Z} / p^{n} \mathbf{Z}$ (where $\mathbf{Z} / p^{n} \mathbf{Z}$ is always viewed as a trivial $G(F)$-module). This isomorphism is not canonical, since it depends on the choice of a generator of $\mu_{p^{n}}$. From the isomorphism $\mathbf{Z} / p^{n} \mathbf{Z} \otimes \mathbf{Z} / p^{n} \mathbf{Z} \cong \mathbf{Z} / p^{n} \mathbf{Z}$ given by ring multiplication, we obtain a noncanonical $G(F)$-module mapping $\mu_{p^{n}} \otimes \mu_{p^{n}} \rightarrow \mu_{p^{n}}$ which induces the cupproduct pairing

$$
\cup: H^{1}\left(G(F), \mu_{p^{n}}\right) \times H^{1}\left(G(F), \mu_{p^{n}}\right) \rightarrow H^{2}\left(G(F), \mu_{p^{n}}\right) .
$$

Recall (cf. [T, (4.2), p. 266]) that under the second isomorphism in (1.2) (a) $\cup(b) \in$ $H^{2}\left(G(F), \mu_{p^{n}}\right)$ corresponds to the Brauer class of the symbol $A_{\omega}(a, b ; F)$, where $\omega$ is the generator of $\mu_{p^{n}}$ mapped to 1 in $\mathbf{Z} / p^{n} \mathbf{Z}$. We will need to use the powerful theorem of Merkurjev and Suslin [MS, Theorem 11.5]:

ThEOREM 1.3 (MERKURJEV - SUSLIN). Let $F$ be an field with $\mu_{p^{n}} \subseteq F$ (so char $F \neq$ p). Then there is a short exact sequence

$$
0 \rightarrow S \rightarrow H^{1}\left(G(F), \mu_{p^{n}}\right) \otimes H^{1}\left(G(F), \mu_{p^{n}}\right) \rightarrow H^{2}\left(G(F), \mu_{p^{n}}\right) \rightarrow 0,
$$


where $S$ is the Steinberg relation group of $F$, i.e., the subgroup of $\otimes_{i=1}^{2} H^{1}\left(G(F), \mu_{p^{n}}\right)$ generated by $\left\{(a) \otimes(1-a) \mid a \in F^{*}, a \neq 1\right\}$.

In this exact sequence the map into $H^{2}\left(G(F), \mu_{p^{\prime \prime}}\right)$ is the cup product. Of course, the surjectivity of this map says that $p^{n} \operatorname{Br}(F)$ is generated by cyclic algebras whenever $\mu_{p^{n}} \subseteq F$.

Because of the need to work with pro-p-groups, we will use a $p$-version of Galois cohomology, which we now describe. For any field $F$ with $\operatorname{char} F \neq p$, let $F(p)$ denote the p-closure of $F$, which is the compositum in $\tilde{F}$ of all the Galois extensions $K$ of $F$ with $[K: F]$ a power of $p$. Then $\mathscr{G}(F(p) / F)$ is a pro-p-group. Since every maximal proper subgroup of a finite $p$-group is normal of index $p$, we have,

If $F \subseteq L \subseteq F(p)$ and $[L: F]<\infty$, then there is a chain of fields $F=K_{0} \subseteq K_{1} \subseteq \cdots \subseteq K_{m}=L$, such that $\left[K_{i}: K_{i-1}\right]$ $=p$ and $K_{i}$ is Galois over $K_{i-1}$ for $i=1,2, \ldots, m$.

In $\S 6$ we will work with fields $F$ with $\mu_{p} \nsubseteq F$. When this occurs it is desirable to work with a somewhat larger extension than $F(p)$, but one which agrees with $F(p)$ when $\mu_{p} \subseteq F$. We define the pth root closure of $F$, denoted $\tilde{F}_{p}$, to be $F\left(\mu_{p}\right)(p)$. From Kummer theory we see

$$
\tilde{F}_{p}=\bigcup_{i=1}^{\infty} K_{i}, \quad \text { where } K_{0}=F \text { and } K_{i+1}=K_{i}\left(\left\{c^{1 / p} \mid c \in K_{i}\right\}\right) .
$$

Clearly $\tilde{F}_{p}$ is Galois over $F$ and $\mathscr{G}\left(\tilde{F}_{p} / F\right)$ is pro-solvable, though not a pro-p-group when $\mu_{p} \nsubseteq F$. Since $\tilde{F}_{p}^{p}=\tilde{F}_{p}$ and $\mu_{p^{n}} \subseteq \tilde{F}_{p}$, the Merkurjev-Suslin theorem shows that $\operatorname{Br}\left(\tilde{F}_{p}\right)$ has no $p$-primary torsion. By contrast, it is unknown whether $\operatorname{Br}_{p}(F(p))$ $=(0)$ when $\mu_{p} \nsubseteq F$.

We will use the notation $G_{p}(F)$ for $\mathscr{G}\left(\tilde{F}_{p} / F\right)$. For any discrete $G_{p}(F)$-module $M$, we write $H_{p}^{i}(F, M)$ for $H^{i}\left(G_{p}(F), M\right)$; we call $H_{p}^{*}(F,-)$ the $p$-Galois cohomology of $F$. When $p=2$ this coincides with the quadratic cohomology $H_{q}^{*}(F,-)$ considered in [AEJ]. (For whenever $\operatorname{char} F \neq 2, \mu_{2} \subseteq F$, so $\tilde{F}_{2}=F(2)$ which is the quadratic closure of $F$.)

Now, $\tilde{F}_{p}^{*}$ is a discrete $G_{p}(F)$-module and we recall $[\mathbf{C F}$, pp. 124-126] that $H_{p}^{0}\left(F, \tilde{F}_{p}^{*}\right) \cong F^{*}, \quad H_{p}^{1}\left(F, \tilde{F}_{p}^{*}\right)=0$ (the homological Hilbert Theorem 90), and $H_{p}^{2}\left(F, \tilde{F}_{p}^{*}\right) \cong \operatorname{Br}\left(\tilde{F}_{p} / F\right):=\operatorname{ker}\left(\operatorname{Br}(F) \rightarrow \operatorname{Br}\left(\tilde{F}_{p}\right)\right)$. Following the same route that led to formulas (1.2), we consider the short exact sequence of $G_{p}(F)$-modules

$$
1 \rightarrow \mu_{p^{n}} \rightarrow \tilde{F}_{p}^{*} \stackrel{p^{n}}{\rightarrow} \tilde{F}_{p}^{*} \rightarrow 1,
$$

where the right-hand map is $a \mapsto a^{p^{n}}$. In view of the description just given of $H_{p}^{i}\left(F, \tilde{F}_{p}^{*}\right)$, the long exact sequence in cohomology obtained from (1.6) begins

$$
\begin{aligned}
0 & \rightarrow \mu_{p^{n}} \rightarrow F^{*} \stackrel{p^{n}}{\rightarrow} F^{*} \rightarrow H^{1}\left(F, \mu_{p^{n}}\right) \rightarrow 0 \\
& \rightarrow 0 \rightarrow H^{2}\left(F, \mu_{p^{n}}\right) \rightarrow \operatorname{Br}\left(\tilde{F}_{p} / F\right) \stackrel{p^{n}}{\rightarrow} \operatorname{Br}\left(\tilde{F}_{p} / F\right) \rightarrow \cdots .
\end{aligned}
$$

Thus, we find,

$$
H_{p}^{1}\left(F, \mu_{p^{n}}\right) \cong F^{*} / F^{* p^{n}} \quad \text { and } \quad H_{p}^{2}\left(F, \mu_{p^{n}}\right) \cong_{p^{n}} \operatorname{Br}(F) .
$$


The second isomorphism in (1.7) uses the nontrivial fact noted above that $\operatorname{Br}_{p}\left(\tilde{F}_{p}\right)=$ 0 , which implies that ${ }_{p^{n}} \operatorname{Br}(F) \subseteq \operatorname{Br}\left(\tilde{F}_{p} / F\right)$. By comparing (1.7) with (1.2) we see that the canonical inflation map $H_{p}^{i}\left(F, \mu_{p^{n}}\right) \rightarrow H^{i}\left(G(F), \mu_{p^{n}}\right)$ must be an isomorphism, $i=1$, 2. Consequently, the Merkurjev-Suslin Theorem 1.3 remains valid when we replace $H^{i}\left(G(F), \mu_{p^{n}}\right)$ by $H_{p}^{i}\left(F, \mu_{p^{n}}\right), i=1,2$.

We conclude this section with some valuation theory, in particular valuation theory relative to the field extension $\tilde{F}_{p} / F$. Let $G$ be an ordered abelian group, written additively, and let $v: F^{*} \rightarrow G$ be a Krull valuation on the field $F$. We will use the following notation: $\Gamma_{F}$ for the value group of $v ; V_{F}$ for the valuation ring of $v ; M_{F}$ for the unique maximal ideal of $V_{F} ; U_{F}$ for the group of units of $V_{F}$; and $\bar{F}$ for the residue field $V_{F} / M_{F}$ of $V_{F}$. For $a \in V_{F}$, we write $\bar{a}$ for the image of $a$ in $\bar{F}$. Usually we will be considering only one valuation at a time on a given field $F$, but when there is more than one we avoid ambiguity by writing $\Gamma_{F, v}, \ldots, U_{F, v}, \bar{F}_{v}$. Good references for valuation theory are $\left[\mathbf{E}\right.$ and $\left.\mathbf{B o}_{2}\right]$.

If $v$ is a valuation on $F$, we say that $v$ is pth root Henselian if $\operatorname{char} \bar{F} \neq p$ and $v$ has a unique extension to $\tilde{F}_{p}$. (This is an example of the $\Omega$-Henselian valuations considered in $[\mathbf{B r}]$, with $\Omega=\tilde{F}_{p}$. From Bröcker's observations we have that $v$ is $p$ th root Henselian just when $\operatorname{char} \bar{F} \neq p$ and Hensel's lemma applies to all monic polynomials $f \in V_{F}[X]$ which split in $\tilde{F}_{p}$. See [Br, (1.2)].) When $\mu_{p} \subseteq F$ and char $\bar{F} \neq p$, a $p$ th root Henselian valuation is the same as a $p$-Henselian valuation as considered in $\left[\mathbf{W}_{1}, \S 1\right]$, and we then use the terms " $p$-Henselian" and " $p$ th root Henselian" interchangeably. The following easy lemma was proved in $\left[\mathbf{W}_{\mathbf{1}},(1.2)\right.$, (1.4)].

Lemma 1.8. Let $(F, v)$ be a valued field with $\mu_{p} \subseteq F$ and $\operatorname{char} \bar{F} \neq p$. Then,

(i) $v$ is $p$-Henselian iff $1+M_{F} \subseteq F^{* p}$;

(ii) if $v$ is $p$-Henselian, then $U_{F} / U_{F}^{p^{n}} \cong \bar{F}^{*} / \bar{F}^{* p^{n}}$; hence

$$
F^{*} / F^{* p^{n}} \cong\left(\bar{F}^{*} / \bar{F}^{* p^{n}}\right) \oplus\left(\Gamma_{F} / p^{n} \Gamma_{F}\right) .
$$

For any valued field $(F, v)$ with $\operatorname{char} \bar{F} \neq p$ we can construct the pth root Henselization of $(F, v)$ by a process analogous to the construction of the usual Henselization, as in [E, pp. 131-132]: Let $w$ be any extension of $v$ to $\tilde{F}_{p}$, and let $K$ be the fixed field of the decomposition group of $\left(\tilde{F}_{p}, w\right)$ over $(F, v)$. The $p$ th root Henselization of $(F, v)$ is defined to be $\left(K,\left.w\right|_{K}\right)$. It is easy to see that $\left(K,\left.w\right|_{K}\right)$ is $p$ th root Henselian and is an immediate extension of $(F, v)$. Note that the $p$ th root Henselization is, up to isomorphism, independent of the choice of $w$.

In analogy with the terminology of algebraic geometry we will call a valued field $(F, v)$ strictly $p$-Henselian if it is $p$ th root Henselian and $\bar{F}=\tilde{F}_{p}$. Let $\hat{\mathbf{Z}}_{p}$ $=\lim _{n} \mathbf{Z} / p^{n} \mathbf{Z}$, the $p$-adic integers, which is the free abelian pro-p-group of rank 1 .

Lemma 1.9. Suppose $(F, v)$ is strictly p-Henselian. Then

(i) $\mu_{p^{n}} \subseteq F$ for all $n$;

(ii) $U_{F} \subseteq F^{* p}$;

(iii) $F^{*} / F^{* p^{n}} \cong \Gamma_{F} / p^{n} \Gamma_{F}$;

(iv) if $\operatorname{dim}_{\mathbf{z} / p} \mathbf{z}\left(\Gamma_{F} / p \Gamma_{F}\right)=m$, then $G_{p}(F) \cong \oplus_{i=1}^{m} \hat{\mathbf{z}}_{p}$. 
Proof. Because $\mu_{p} \subseteq \bar{F}$ and $v$ extends uniquely to $F\left(\mu_{p}\right) \subseteq \tilde{F}_{p}$ we must have $\mu_{p} \subseteq F$. The rest of (i)-(iii) follow easily from Lemma 1.8. If $K \supseteq F$ is any finite degree Galois extension of $F$ with $K \subseteq \tilde{F}_{p}$, then $[K: F]=p^{k}, v$ extends uniquely to $K$ and $\tilde{\bar{F}}_{p}=\bar{F} \subseteq \bar{K} \subseteq \overline{\tilde{F}}_{p}=\tilde{\bar{F}}_{p}$. So, $\bar{K}=\bar{F}$. Since char $\bar{F}+[K: F]$, the argument of [S, p. 66] shows that $K$ is a Kummer extension of $F$. Thus, by Kummer theory,

$$
\tilde{F}_{p}=\bigcup_{n=1}^{\infty} F_{n}, \quad \text { where } F_{n}=F\left(\left\{c^{1 / p^{n}} \mid c \in F^{*}\right\}\right) \text {. }
$$

Because $\Gamma_{F}$ is a torsion-free abelian group, any inverse image of a $\mathbf{Z} / p \mathbf{Z}$-base of $\Gamma_{F} / p \Gamma_{F}$ is a base of $\Gamma_{F} / p^{n} \Gamma_{F}$ as a free $\mathbf{Z} / p^{n} \mathbf{Z}$-module. By Kummer theory and (iii), $\mathscr{G}\left(F_{n} / F\right) \cong F^{*} / F^{* p^{n}} \cong\left(\mathbf{Z} / p^{n} \mathbf{Z}\right)^{m}$. Consequently,

$$
G_{p}(F)=\lim _{\leftarrow} \mathscr{G}\left(F_{n} / F\right) \cong\left(\hat{\mathbf{Z}}_{p}\right)^{m}
$$

as desired.

For any valued field $(F, v)$ with char $\bar{F} \neq p$, let $w$ be an extension of $v$ to $\tilde{F}_{p}$. Let $L$ be the fixed field of the inertia group of $\left(\tilde{F}_{p}, w\right)$ over $(F, v)$. We call $\left(L,\left.w\right|_{L}\right)$ the strict $p$-Henselization of $(F, v)$. Note $\left(L,\left.w\right|_{I}\right)$ is strictly $p$-Henselian and is a maximal unramified extension of $(F, v)$ in $\tilde{F}_{p}$. The strict $p$-Henselization is unique up to isomorphism. (Similarly, we obtain a "strict Henselization" of $(F, v)$ by replacing $\tilde{F}_{p}$ by $\tilde{F}$ in this construction.)

2. Valuation theory of division algebras. In this section we will give a construction for obtaining valued division algebras, and we will show how a valuation on a division algebra can restrict the possible Galois groups over the center of maximal subfields.

Let $D$ be a division algebra and let $D^{*}=D-\{0\}$. A valuation $v$ on $D$ is a function $v: D^{*} \rightarrow \Gamma$ (where $\Gamma$ is a totally ordered group), such that for all $a$, $b \in D^{*}$,

(i) $v(a b)=v(a)+v(b)$;

(ii) $v(a+b) \geqslant \min (v(a), v(b))$ if $b \neq-a$.

We use the same notation as with fields for the objects associated to $v$ : the value group of $v$ is $\Gamma_{D}=v\left(D^{*}\right)$; the valuation ring of $D$ is $V_{D}=\left\{a \in D^{*} \mid v(a) \geqslant 0\right\} \cup$ $\{0\}$; the unique maximal left ideal and unique maximal right ideal of $V_{D}$ is $M_{D}=\left\{a \in D^{*} \mid v(a)>0\right\} \cup\{0\}$; the residue division ring is $\bar{D}=V_{D} / M_{D}$; and the group of units of $V_{D}$ is $U_{D}=V_{D}-M_{D}$. We will consider only division algebras finite dimensional over their centers; for such a $D$, with center $F, \Gamma_{F}$ is central in $\Gamma_{D}$ and $\Gamma_{D} / \Gamma_{F}$ is torsion. Hence $\Gamma_{D}$ must be abelian, justifying our additive notation for it. The standard reference for valued division algebras is Schilling's book [S].

Let $E$ be a subdivision algebra of the valued division algebra $(D, v)$, and suppose $[D: E]<\infty$, where $[D: E]$ denotes the dimension of $D$ as a right $E$ vector space. Then the restriction $\left.v\right|_{E}$ of $v$ to $E^{*}$ is a valuation on $E$. Recall [S, p. 21] that the following version of the "fundamental inequality" holds for the extension $v$ over $\left.v\right|_{E}$ :

$$
[\bar{D}: \bar{E}] \cdot\left|\Gamma_{D}: \Gamma_{E}\right| \leqslant[D: E]
$$


We say that $v$ is totally ramified over $\left.v\right|_{E}$ if $\left|\Gamma_{D}: \Gamma_{E}\right|=[D: E]$. Then, of course, $\bar{D}=\bar{E}$.

The next proposition and its corollaries provide the link between Galois groups and value groups of division algebras. The proposition is well known (cf. [E, (20.11) (d), (20.18); S, p. 66, p. 86, Remark 1]), but we sketch a proof since it is vital for our examples.

Proposition 2.2. Let $F \subseteq K$ be fields with $[K: F]<\infty$ and $K$ Galois over $F$. Suppose $K$ has a valuation $v$ which is totally ramified over $\left.v\right|_{F}$, and suppose char $\bar{K}+$ $[K: F]$. Then $\mathscr{G}(K / F) \cong \Gamma_{K} / \Gamma_{F}$, and $\bar{F}$ contains a primitive lth root of unity, where $l$ is the exponent of the abelian group $\Gamma_{K} / \Gamma_{F}$.

SKeTCH OF THE PROOF. Since $\overline{\sigma(u)}=\bar{u}$ for $u \in U_{K}$, the function $\mathscr{G}(K / F) \times K^{*}$ $\rightarrow \bar{K}^{*}=\bar{F}^{*}$ given by $(\sigma, a) \mapsto \overline{\sigma(a) / a}$ induces a bimultiplicative pairing $\gamma: \mathscr{G}(K / F) \times \Gamma_{K} / \Gamma_{F} \rightarrow \bar{F}^{*}$. The proposition follows easily once it is known that $\gamma$ is nondegenerate. Assume first that $\left(F,\left.v\right|_{F}\right)$ is Henselian with separably closed residue field. Then $K$ is a Kummer extension of $F$ by [S, p. 64, Theorem 3], and the nondegeneracy of $\gamma$ follows from the nondegeneracy of the Kummer pairing. Dropping the restrictions on $F$, let $L$ be a maximal unramified extension of $\left(F,\left.v\right|_{F}\right)$ in $\tilde{F}$. Then $L$ is Henselian with separably closed residue field, and $L$ and $K$ are linearly disjoint over $F$ as $K / F$ is totally ramified. Since $\mathscr{G}(K \cdot L / L) \cong \mathscr{G}(K / F)$ and $\Gamma_{K \cdot L}=\Gamma_{K}, \Gamma_{L}=\Gamma_{F}$, the pairing $\gamma$ for $K$ over $F$ coincides with the corresponding pairing of $K \cdot L$ over $L$, which we have seen to be nondegenerate.

COROllaRy 2.3. Let $D$ be a division algebra finite dimensional over a field $F$. Suppose $D$ has a valuation $v$ totally ramified over $\left.v\right|_{F}$, and suppose char $\bar{F}+[D: F]$. If $K \supseteq F$ is any subfield of $D$ which is Galois over $F$, then $\mathscr{G}(K / F)$ is isomorphic to a subgroup of $\Gamma_{D} / \Gamma_{F}$.

Proof. By the fundamental inequality (2.1) and the transitivity formula for ramification index, $\left.v\right|_{K}$ must be totally ramified over $\left.v\right|_{F}$. Hence, by the proposition, $\mathscr{G}(K / F) \cong \Gamma_{K} / \Gamma_{F} \subseteq \Gamma_{D} / \Gamma_{F}$.

COROllaRY 2.4. Let $(F, v)$ be a valued field with $\operatorname{char} \bar{F} \neq p$ and $\mu_{p} \nsubseteq \bar{F}$ for some prime $p$. Suppose $K$ is a Galois extension field of $F$ with $[K: F]=p^{n}$, and suppose $v$ has a unique extension to a valuation $w$ of $K$. Then $K$ is an inertial extension of $F$ (i.e., $[\bar{K}: \bar{F}]=[K: F])$ and $\mathscr{G}(K / F) \cong \mathscr{G}(\bar{K} / \bar{F})$.

Proof. Let $L$ be the inertia field of $w$ over $F$. Then as $v$ is indecomposed in $K$ and $\bar{K}$ is separable over $\bar{F}$ (since $\operatorname{char} \bar{F}+[K: F]$ and $[\bar{K}: \bar{F}] \mid[K: F]$ ) we have $L / F$ is inertial and Galois, $\bar{L}=\bar{K}, \bar{L} / \bar{F}$ is Galois, and $\mathscr{G}(\bar{L} / \bar{F}) \cong \mathscr{G}(L / F)$ (cf. [E. $\S 19])$. So, it suffices to see that $K=L$. Because $\bar{L}=\bar{K}$ and $\left.w\right|_{L}$ extends uniquely to $K$ and char $\bar{L}+[K: L],(K, w)$ must be totally ramified over $\left(L,\left.w\right|_{L}\right)$. Since $[K: L]$ is a $p$-power, if $K \neq L$ then Proposition 2.2 implies $\mu_{p} \subseteq \bar{L}$. However, $[\bar{L}: \bar{F}]=[L: F]$ is a $p$-power, so $\mu_{p} \nsubseteq \bar{L}$, as $\mu_{p} \nsubseteq \bar{F}$. Hence, $K=L$, as desired.

The next theorem gives the criterion we will use for the existence of a valuation on an algebra. The somewhat cumbersome hypotheses cover the examples both in $\$ 5$ and in §6. Similar theorems will appear in $\left[\mathbf{W}_{2}\right]$. 
THEOREM 2.5. Let $A$ be an algebra finite dimensional over a field, and let $L \subseteq A$ be a field. Let $v$ be a valuation on $L$, and let $\Delta$ be the divisible hull of $\Gamma_{L}$ (so $\left.\Delta \cong \Gamma_{L} \otimes_{\mathbf{Z}} \mathbf{Q}\right)$. Suppose there are elements $a_{1}, \ldots, a_{m}$ of the group of units $A^{*}$ of $A$ satisfying

(i) $\left\{a_{1}, \ldots, a_{m}\right\}$ is a base of $A$ as a right L-vector space;

(ii) for each $l \in L^{*}$ and each $a_{i}, a_{i} l a_{i}^{-1} \in L$ and $v\left(a_{i} l a_{i}^{-1}\right)=v(l)$;

(iii) $a_{i} a_{j} a_{i}^{-1} a_{j}^{-1} \in U_{L}$ (the group of units of $v$ ) for all $i, j$;

(iv) $\mathscr{A}:=\left\{a_{1} L^{*}, \ldots, a_{m} L^{*}\right\}$ is an abelian subgroup of $N_{A^{*}}\left(L^{*}\right) / L^{*}$, where $N_{A^{*}}\left(L^{*}\right)$ is the normalizer of $L^{*}$ in $A^{*}$.

Then there is a well-defined group homomorphism $\bar{w}: \mathscr{A} \rightarrow \Delta / \Gamma_{L}$ given by $a_{i} L^{*} \mapsto \frac{1}{m} v\left(a_{i}^{m}\right)+\Gamma_{L}$. Suppose $\bar{w}$ is injective. Then $v$ extends to a valuation $w$ on $A$; hence $A$ is a division ring. $\Gamma_{A}$ is the subgroup of $\Delta$ such that $\Gamma_{A} / \Gamma_{L}=\bar{w}(\mathscr{A})$. Furthermore, $(A, w)$ is totally ramified over $(L, v)$, and $\bar{A}=\bar{L}$.

Proof. Condition (iv) shows that $a_{i}^{m} \in L^{*}$ for each $i$. Hence, the function $\bar{w}$ is well defined. For any $a_{i}$ and any $c \in L^{*}$ we have the general identity

$$
\left(a_{i} c\right)^{m}=c^{a_{i}} c^{a_{i}^{2}} \ldots c^{a_{i}^{m}} a_{i}^{m}
$$

where $c^{a_{i}}$ means $a_{i} c a_{i}^{-1}$. Hence, by (ii),

$$
v\left(\left(a_{i} c\right)^{m}\right)=m v(c)+v\left(a_{i}^{m}\right) .
$$

Let $T$ be the subgroup of $A^{*}$ generated by $\left\{a_{1}, \ldots, a_{m}\right\}$, and $T^{\prime}$ its commutator subgroup. By (ii), $T U_{L}$ is a group in which $U_{I}$ is a normal subgroup. By (iii), $T U_{L} / U_{L}$ is abelian, so $T^{\prime} \subseteq U_{L}$. Since for any $i, j,\left(a_{i} a_{j}\right)^{m}=a_{i}^{m} a_{j}^{m} t$ with $t \in T^{\prime} \subseteq$ $U_{L}$, we have

$$
v\left(\left(a_{i} a_{j}\right)^{m}\right)=v\left(a_{i}^{m}\right)+v\left(a_{j}^{m}\right) .
$$

From (1) and (2) it follows that $\bar{w}$ is a group homomorphism.

Because $\Delta$ is torsion-free and $\Delta / \Gamma_{L}$ is torsion, the ordering on $\Gamma_{L}$ has a unique extension to $\Delta$ which makes $\Delta$ a totally ordered abelian group. This is the ordering on $\Delta$ we use.

Now suppose $\bar{w}$ is injective. Define a function $w: A-\{0\} \rightarrow \Delta$ as follows: For any $a_{i}$ and any $c \in L^{*}$, set

$$
w\left(a_{i} c\right)=\frac{1}{m} v\left(a_{i}^{m}\right)+v(c) .
$$

Now, for any $\alpha \in A-\{0\}, \alpha$ has a unique representation $\alpha=\sum_{i=1}^{m} a_{i} c_{i}$ with the $c_{i} \in L$, some $c_{i} \neq 0$; define $w(\alpha)=\inf \left\{w\left(a_{i} c_{i}\right) \mid c_{i} \neq 0\right\}$. Since $\bar{w}$ is injective, $w\left(a_{i} c_{i}\right)$ $\neq w\left(a_{j} c_{j}\right)$ for $i \neq j$. Thus, there is a unique summand $a_{j} c_{j}$ of $\alpha$ with $w\left(a_{j} c_{j}\right)=w(\alpha)$; we call $a_{j} c_{j}$ the leading term of $\alpha$. Take any $\beta=\sum a_{i} d_{i} \in A-\{0\}\left(d_{i} \in L\right)$ with $\beta \neq-\alpha$. Let $a_{k}\left(c_{k}+d_{k}\right)$ be the leading term of $\alpha+\beta$. We have, if $c_{k} \neq 0, d_{k} \neq 0$,

$$
\begin{aligned}
w\left(a_{k}\left(c_{k}+d_{k}\right)\right) & =\frac{1}{m} v\left(a_{k}^{m}\right)+v\left(c_{k}+d_{k}\right) \\
& \geqslant \inf \left(\frac{1}{m} v\left(a_{k}^{m}\right)+v\left(c_{k}\right), \frac{1}{m} v\left(a_{k}^{m}\right)+v\left(d_{k}\right)\right) \\
& =\inf \left(w\left(a_{k} c_{k}\right), w\left(a_{k} d_{k}\right)\right) \geqslant \inf (w(\alpha), w(\beta)) .
\end{aligned}
$$


Hence, $w(\alpha+\beta)=w\left(a_{k}\left(c_{k}+d_{k}\right)\right) \geqslant \inf (w(\alpha), w(\beta))$; this inequality still holds if $c_{k}=0$ or $d_{k}=0$. Since $w(-\alpha)=w(\alpha)$, the usual argument shows:

$$
\text { if } w(\alpha) \neq w(\beta) \text {, then } w(\alpha+\beta)=\inf (w(\alpha), w(\beta)) \text {. }
$$

It remains to check that $w(\alpha \beta)=w(\alpha)+w(\beta)$. Take any $a_{i}$ and $a_{j}$ and write $a_{i} a_{j}=a_{k} e$ with $e \in L^{*}$. Then for any $c, d \in L^{*}$,

$$
\begin{aligned}
w\left(a_{i} c a_{j} d\right) & =w\left(a_{k} e\left(a_{j}^{-1} c a_{j}\right) d\right) \\
& =\frac{1}{m} v\left(a_{k}^{m}\right)+v(e)+v\left(a_{j}^{-1} c a_{j}\right)+v(d) \\
& =\frac{1}{m} v\left(\left(a_{k} e\right)^{m}\right)+v(c)+v(d) \text { by (1) and (ii) } \\
& =\frac{1}{m}\left[v\left(a_{i}^{m}\right)+v\left(a_{j}^{m}\right)\right]+v(c)+v(d) \text { by (2) } \\
& =w\left(a_{i} c\right)+w\left(a_{j} d\right) .
\end{aligned}
$$

Now, for any $\alpha=\sum a_{i} c_{i}$ and $\beta=\sum a_{i} d_{i} \in A-\{0\}$ we have

$$
\begin{aligned}
w(\alpha \beta) & =w\left(\sum_{i, l} a_{i} c_{i} a_{l} d_{l}\right) \geqslant \inf _{i, l}\left\{w\left(a_{i} c_{i} a_{l} d_{l}\right) \mid c_{i}, d_{l} \neq 0\right\} \\
& =\inf _{i, l}\left\{w\left(a_{i} c_{i}\right)+w\left(a_{l} d_{l}\right) \mid c_{i}, d_{l} \neq 0\right\} \geqslant w(\alpha)+w(\beta) .
\end{aligned}
$$

Say $a_{j} c_{j}$ is the leading term of $\alpha$, and set $\alpha^{\prime}=\alpha-a_{j} c_{j}$. So, $w(\alpha)=w\left(a_{j} c_{j}\right)<w\left(\alpha^{\prime}\right)$ (or $\alpha^{\prime}=0$ ). Likewise, set $\beta^{\prime}=\beta-a_{k} d_{k}$, where $a_{k} d_{k}$ is the leading term of $\beta$. Then,

$$
\alpha \beta=\left(a_{j} c_{j} a_{k} d_{k}\right)+\alpha^{\prime}\left(a_{k} d_{k}\right)+\left(a_{j} c_{j}\right) \beta^{\prime}+\alpha^{\prime} \beta^{\prime} .
$$

By (4) and (5) the first summand here has value strictly smaller than the other three. Hence, by (3) and (4), $\alpha \beta \neq 0$ and

$$
w(\alpha \beta)=w\left(a_{j} c_{j} a_{k} d_{k}\right)=w\left(a_{j} c_{j}\right)+w\left(a_{k} d_{k}\right)=w(\alpha)+w(\beta) .
$$

Since we have just seen that the finite dimensional algebra $A$ has no zero divisors, it must be a division algebra. Our calculations show that $w: A-\{0\} \rightarrow \Delta$ is a valuation on $A$. It is easy to check that $\left.w\right|_{L}=v$.

Clearly the value group $\Gamma_{A}$ is the subgroup of $\Delta$ generated by $\left\{w\left(a_{1}\right), \ldots, w\left(a_{m}\right)\right\}$ and $\Gamma_{L}$; so $\Gamma_{A} / \Gamma_{L}=\operatorname{im}(\bar{w})$. As $\bar{w}$ is injective, $\left|\Gamma_{A} / \Gamma_{L}\right|=|\mathscr{A}|=m=[A: L]$, i.e. $A$ is totally ramified over $L$. Take any $\alpha=\sum a_{i} c_{i} \in A^{*}$ with $w(\alpha)=0$. If the leading term of $\alpha$ is $a_{j} c_{j}$, we have $w\left(a_{j} c_{j}\right)=w(\alpha)=0$. Since $w\left(a_{j}\right)=-w\left(c_{j}\right) \in \Gamma_{L}$, the injectivity of $\bar{w}$ implies $a_{j} \in L^{*}$; so $a_{j} c_{j} \in U_{L}$. Hence, in $\bar{A}, \bar{\alpha}=\overline{a_{j} c_{j}} \in \bar{L}$. Thus, $\bar{A}=\bar{L}$.

Corollary 2.6. Consider the algebra

$$
A=A_{\omega_{1}}\left(b_{1}, c_{1} ; F\right) \otimes_{F} \cdots \otimes_{F} A_{\omega_{k}}\left(b_{k}, c_{k} ; F\right),
$$

where $\omega_{m}$ is a primitive $n_{m}$ th root of unity in a field $F$ and $b_{1}, c_{1}, \ldots, b_{k}, c_{k} \in F^{*}$. Let $n=n_{1} \cdots n_{k}$ and $l=\operatorname{lcm}\left(n_{1}, \ldots, n_{k}\right)$. Let $v$ be $a$ valuation on $F$. Suppose $\left\{\left(l / n_{m}\right) v\left(b_{m}\right),\left(l / n_{m}\right) v\left(c_{m}\right) \mid 1 \leqslant m \leqslant k\right\}$ generates a subgroup of order $n^{2}$ in $\Gamma_{F} / l \Gamma_{F}$. 
Then $A$ is a division algebra and $v$ extends to a valuation on $A$ totally ramified over $F$, with $\bar{A}=\bar{F}$ and with value group $\Gamma_{A}$ generated by $\left\{\left(1 / n_{m}\right) v\left(b_{m}\right),\left(1 / n_{m}\right) v\left(c_{m}\right) \mid 1 \leqslant\right.$ $m \leqslant k\}$ and $\Gamma_{F}$. So,

$$
\Gamma_{A} / \Gamma_{F} \cong \prod_{m=1}^{k}\left(\mathbf{Z} / n_{m} \mathbf{Z} \times \mathbf{Z} / n_{m} \mathbf{Z}\right)
$$

Proof. For $1 \leqslant m \leqslant k$, let $i_{m}, j_{m} \in A$ be the standard generators of

$$
A_{\omega_{m}}\left(b_{m}, c_{m} ; F\right) \text {. }
$$

We want to apply the theorem with $L=F$ and the $a_{i}$ all being products $i_{1}^{r_{1}} j_{1}^{s_{1}} \ldots$ $i_{k}^{r_{k}} j_{k}^{s_{k}}$ with $0 \leqslant r_{m}<n_{m}, 0 \leqslant s_{m}<n_{m}$, for $1 \leqslant m \leqslant k$. There are $n^{2}$ of the $a_{i}$ and they clearly form an $F$-base of $A$. Every commutator $a_{i} a_{j} a_{i}^{-1} a_{j}^{-1}$ is a product of roots of unity. Condition (ii) holds trivially since $F$ is the center of $D$. It follows easily that (i)-(iv) of the theorem all hold. We have $\bar{w}\left(i_{m} F^{*}\right)=\left(1 / n_{m}\right) v\left(b_{m}\right)+\Gamma_{F}$ and $\bar{w}\left(j_{m} F^{*}\right)=\left(1 / n_{m}\right) v\left(c_{m}\right)+\Gamma_{F}$. The assumption on the values of the $b_{m}$ and $c_{m}$ implies that $\bar{w}(\mathscr{A})$ is a subgroup of order $n^{2}$ of $(1 / l) \Gamma_{F} / \Gamma_{F}$. Hence, $\bar{w}$ must be injective, and the corollary follows from the theorem.

EXAMPLE 2.7. Let $K$ be a field containing a primitive $p^{r}$ th root of unity $\omega$ for some prime $p$. Let $z_{1}, \ldots, z_{2 l}$ be independent commuting indeterminates over $K$, and let $F=K\left(z_{1}, \ldots, z_{2 l}\right)$. The lexicographic ordering makes $\Gamma:=\prod_{i=1}^{2 l} \mathbf{Z}$ into a totally ordered abelian group. There is a unique valuation $v: F^{*} \rightarrow \Gamma$ such that $v\left(z_{i}\right)=(0, \ldots, 0,1,0, \ldots, 0)$ (the 1 in the $i$ th place) for each $i$ and $v(c)=0$ for all $c \in K^{*}$. Specifically, $v$ is defined first on $K\left[z_{1}, \ldots, z_{2 l}\right]-\{0\}$ by

$$
v\left(\sum_{i_{1}} \cdots \sum_{i_{2 l}} c_{i_{1} i_{2} \cdots i_{2 l}} z_{1}^{i_{1}} \cdots z_{2 l}^{i_{2 l}}\right)=\inf \left\{\left(i_{1}, \ldots, i_{2 l}\right) \mid c_{i_{1}} \cdots i_{2 l} \neq 0\right\} .
$$

Then $v$ is extended to the quotient field $F$ by defining $v(a / b)=v(a)-v(b)$ for all $a, b \in K\left[z_{1}, \ldots, z_{2 l}\right]-\{0\}$. Let $A=A_{\omega}\left(z_{1}, z_{2} ; F\right) \otimes_{F} \cdots \otimes_{F} A_{\omega}\left(z_{2 l-1}, z_{2 l} ; F\right)$. By the corollary, $A$ is a division algebra and $v$ extends to a valuation on $A$ which is totally ramified over $F$, with $\Gamma_{A} / \Gamma_{F} \cong\left(\mathbf{Z} / p^{r} \mathbf{Z}\right)^{2 l}$ and $\bar{A}=\bar{F}=K$. Hence, by Corollary 2.3, if $K \supseteq F$ is any subfield of $A$ which is Galois over $F$, then $\mathscr{G}(K / F)$ is isomorphic to a subgroup of $\left(\mathbf{Z} / p^{r} \mathbf{Z}\right)^{2 l}$. Note also that if $L \supseteq F$ is any field which has an unramified extension of $v$, then Corollary 2.6 applies equally well to $A \otimes_{F} L$. So, $A \otimes_{F} L$ is also a valued division algebra, with $\Gamma_{A \otimes L} / \Gamma_{L} \cong \Gamma_{A} / \Gamma_{F}$.

REMARK 2.8. For any prime $p$, let $k$ be any field with char $k \neq p$, and let $K=k\left(\mu_{p^{\prime \prime}}\right)$ for some fixed $n \geqslant 3$. If we let $r=1$ and $l=n$ in the preceding example, then we obtain a division algebra $D_{1}$ of index $p^{n}$ in which every maximal subfield of $D_{1}$ Galois over its center has an elementary abelian Galois group of order $p^{n}$. If we let $r=n$ and $l=1$ in the example we obtain another division algebra $D_{2}$ of index $p^{n}$. The Galois group over the center of $D_{2}$ of any Galois maximal subfield must be a subgroup of $\left(\mathbf{Z} / p^{n} \mathbf{Z}\right) \times\left(\mathbf{Z} / p^{n} \mathbf{Z}\right)$ of order $p^{n}$. Such a group cannot be elementary abelian, as $n \geqslant 3$. Since no group can occur as a Galois group of a maximal subfield for both $D_{1}$ and $D_{2}$, it follows by Amitsur's argument (cf. [Am, pp. 418-419; or Ja, p. 93, Theorem 4]) that the generic division algebra 
$U D\left(k, p^{n}\right)$ of index $p^{n}(n \geqslant 3)$ over $k$ is not a crossed product. Amitsur proved this in his original paper (for char $k=0$, and Schacher-Small [SS] did the case char $k=$ prime $\neq p$ ). Amitsur did not mention valued division algebras, but the valuation theory given here applies to (and perhaps clarifies) his examples, which were iterated twisted Laurent power series division algebras.

We now give one more corollary to Theorem 2.5 , which will apply to the examples in $§ 6$.

Corollary 2.9. Let $F$ be a field with valuation $v$. Let $K_{1}, \ldots, K_{k}$ be cyclic Galois extensions of $F$, and let $A=A\left(K_{1} / F, \sigma_{1}, b_{1}\right) \otimes_{F} \cdots \otimes_{F} A\left(K_{k} / F, \sigma_{k}, b_{k}\right)$ for some $b_{1}, \ldots, b_{k} \in F^{*}$. Let $\left[K_{i}: F\right]=n_{i}, n=n_{1} \cdots n_{k}$, and $l=\operatorname{lcm}\left(n_{1}, \ldots, n_{k}\right)$. Suppose

(i) each $K_{i}$ is an inertial extension of $(F, v)$, i.e., $v$ extends (uniquely) to a valuation $v_{i}$ on $K$ with residue field $\bar{K}_{i}$ such that $\left[\bar{K}_{i}: \bar{F}\right]=\left[K_{i}: F\right]$;

(ii) $\bar{K}_{1}, \ldots, \bar{K}_{k}$ are all linearly disjoint over $\bar{F}$;

(iii) $\left\{\left(l / n_{i}\right) v\left(b_{i}\right) \mid 1 \leqslant i \leqslant k\right\}$ generates a subgroup of order $n$ in $\Gamma_{F} / l \Gamma_{F}$.

Then $A$ is a division algebra and $v$ extends to a valuation $w$ on $A$ with residue division algebra $\bar{A}=\bar{K}_{i} \cdots \cdots \bar{K}_{k}$ and value group $\Gamma_{A}$ generated by $\left\{\left(1 / n_{i}\right) v\left(b_{i}\right)\right\}$ and $\Gamma_{F}$. So, $[\bar{A}: \bar{F}]=\left|\Gamma_{A}: \Gamma_{F}\right|=n$.

Proof. Let $L$ be the compositum $K_{1} \cdots K_{k}$ in $\tilde{F}$, and let $u$ be any extension of $v$ to $L$, with $\bar{L}$ the residue field of $u$. Since $\left.u\right|_{K_{l}}=v_{i}$ by (i), we have each $\bar{K}_{i} \subseteq \bar{L}$. Then from (2.1), (ii), and (i),

$$
[L: F] \geqslant[\bar{L}: \bar{F}] \geqslant\left[\bar{K}_{1} \cdots \cdot \bar{K}_{k}: \bar{F}\right]=\prod_{i=1}^{k}\left[\bar{K}_{i}: \bar{F}\right]=\prod_{i=1}^{k}\left[K_{i}: F\right] \geqslant[L: F] .
$$

So equality must hold throughout. Hence, $L$ is an inertial extension of $F, \bar{L}=$ $\bar{K}_{1} \cdots \bar{K}_{k}$ and $K_{1}, \ldots, K_{k}$ are all linearly disjoint over $F$, so $L \cong K_{1} \otimes_{F}$ $\cdots \otimes_{F} K_{k}$.

Let $x_{j} \in A$ be the standard generator of $A\left(K_{j} / F, \sigma_{j}, b_{j}\right)$ over $K_{j}$. We will apply Theorem 2.5 , taking for the $a_{i}, 1 \leqslant i \leqslant n$, all products $x_{1}^{r_{1}} \cdots x_{k}^{r_{k}}$ with $0 \leqslant r_{j}<n_{j}$ for each $j$. For $L$ we take $K_{1} \otimes_{F} \cdots \otimes_{F} K_{k} \subseteq A$ which we have seen has a unique extension of $v$ on $F$. Clearly the $a_{i}$ form an $L$-base of $A$. Condition (ii) of Theorem 2.5 holds since the $a_{i}$ conjugate each $K_{j}$ to itself and the extension of $v$ to $L$ is unique. Condition (iii) holds trivially because $a_{i} a_{j}=a_{j} a_{i}$, all $i, j$, and (iv) also clearly holds. We have $\bar{w}\left(x_{j} L^{*}\right)=\left(1 / n_{j}\right) v\left(b_{j}\right)+\Gamma_{L}$, and $\Gamma_{L}=\Gamma_{F}$ as $L / F$ is inertial. Assumption (iii) implies that $\bar{w}(\mathscr{A})$ is a subgroup of order $n$ in $(1 / l) \Gamma_{L} / \Gamma_{L}$. Since $|\mathscr{A}|=n, \bar{w}$ must be injective. The conclusions of the corollary follow from the theorem and the observations above about $L$.

3. Cohomology of free abelian pro-p-groups. For any prime number $p$ and any natural number $m$, let $P_{m}:=\bigoplus_{i=1}^{m} \hat{\mathbf{Z}}_{p}$, which is the free abelian pro- $p$-group of rank $m$. As we saw in Lemma 1.9(iv) this group arises as $G_{p}(F)$ for a strictly $p$-Henselian valued field if $\Gamma_{F} / p \Gamma_{F}$ has rank $m$. In this section we prove a result on splitting of elements of $H^{2}\left(P_{m}, \mathbf{Z} / p \mathbf{Z}\right)$ by subgroups of $P_{m}$. We will give purely cohomological arguments, although other approaches are possible. We always consider $\mathbf{Z} / p \mathbf{Z}$ as a trivial $P_{m}$-module. Then $H^{1}\left(P_{m}, \mathbf{Z} / p \mathbf{Z}\right) \cong \operatorname{Hom}\left(P_{m}, \mathbf{Z} / p \mathbf{Z}\right) \cong(\mathbf{Z} / p \mathbf{Z})^{m}$, which will be viewed as a vector space over $\mathbf{Z} / p \mathbf{Z}$. 
Lemma 3.1. Suppose $\chi_{1}, \chi_{2}, \ldots, \chi_{2 t}$ are linearly independent in $H^{1}\left(P_{m}, \mathbf{Z} / p \mathbf{Z}\right)$. Then,

$$
\chi_{1} \cup \chi_{2}+\chi_{3} \cup \chi_{4}+\cdots+\chi_{2 t-1} \cup \chi_{2 t} \neq 0 \quad \text { in } H^{2}\left(P_{m}, \mathbf{Z} / p \mathbf{Z}\right)
$$

PROOF. We verify this by direct calculation, using additive notation for the group operation on $P_{m}$. The cohomology class in question is represented by the 2-cocycle

$$
z(r, s)=\chi_{1}(r) \chi_{2}(s)+\chi_{3}(r) \chi_{4}(s)+\cdots+\chi_{2 t-1}(r) \chi_{2 t}(s),
$$

as the group action is trivial (cf. [Sh, p. 38]). Clearly $z(r, 0)=z(0, s)=0$. If $z$ is a coboundary, there is a continuous function $f: P_{m} \rightarrow \mathbf{Z} / p \mathbf{Z}$ with $z(r, s)=f(s)-$ $f(r+s)+f(r)$. So $f(0)=z(0,0)=0$. Now, choose $a_{1}, a_{2} \in P_{m}$ with $\chi_{i}\left(a_{j}\right)=\delta_{i j}$ (Kronecker delta), $i=1, \ldots, 2 t, j=1,2$. We calculate: $f\left(a_{1}\right)+f\left(-a_{1}\right)=z\left(a_{1},-a_{1}\right)$ $=0$. Thus, as $P_{m}$ is abelian,

$$
\begin{aligned}
f\left(a_{2}\right) & =f\left(a_{1}+a_{2}+-a_{1}\right)=f\left(a_{1}+a_{2}\right)-z\left(a_{1}+a_{2},-a_{1}\right)+f\left(-a_{1}\right) \\
& =f\left(a_{1}\right)-z\left(a_{1}, a_{2}\right)+f\left(a_{2}\right)-0-f\left(a_{1}\right)=-1+f\left(a_{2}\right) .
\end{aligned}
$$

This contradiction proves the lemma.

REMARK 3.2. For an alternative proof observe that Lemma 3.1 is an immediate consequence of the isomorphism $H^{2}\left(P_{m}, \mathbf{Z} / p \mathbf{Z}\right) \cong H^{1}\left(P_{m}, \mathbf{Z} / p \mathbf{Z}\right) \wedge H^{1}\left(P_{m}, \mathbf{Z} / p \mathbf{Z}\right)$ (exterior product) which can be verified by induction on $m$ using the Künneth formula. The lemma is also deducible from Corollary 2.6.

Remark 3.3. Suppose $\Delta \subseteq P_{m}$ is a subgroup of index $p$. Then $\Delta \supseteq p P_{m}$. Using the fact that every lift of a $\mathbf{Z} / p \mathbf{Z}$-base of $P_{m} / p P_{m}$ is a base of $P_{m}$ as a free abelian pro-p-group, it is easy to see that $\Delta \cong P_{m}$ as profinite groups. It follows by induction that for any subgroup $H$ of finite index in $P_{m}, H$ is open in $P_{m}$ and $H \cong P_{m}$.

The main result of this section is needed for the study of splitting fields of the examples in $\$ 5$. What we need is obtainable by adapting to the strictly $p$-Henselian situation the following theorem of Tignol and Amitsur [TA]: If $D$ is a central simple division algebra over a field $F$ with strictly Henselian valuation and $\operatorname{char} \bar{F}+[D: F]$, then every splitting field of $D$ algebraic over $F$ contains a maximal subfield of $D$. We prefer to give an entirely cohomological formulation and proof. For algebras of prime exponent the Tignol-Amitsur theorem is actually deducible from our next theorem.

THEOREM 3.4. Suppose $\chi_{1}, \chi_{2}, \ldots, \chi_{2 t} \in H^{1}\left(P_{m}, \mathbf{Z} / p \mathbf{Z}\right)$ are linearly independent, and set $\gamma=\chi_{1} \cup \chi_{2}+\chi_{3} \cup \chi_{4}+\cdots+\chi_{2 t-1} \cup \chi_{2 t} \in H^{2}\left(P_{m}, \mathbf{Z} / p \mathbf{Z}\right)$. Let $N$ be an open subgroup of $P_{m}$ with $\operatorname{res}_{P_{m} \rightarrow N}(\gamma)=0$. Then, there is a base $\psi_{1}, \ldots, \psi_{2 t}$ of $\operatorname{span}\left\{\chi_{1}, \ldots, \chi_{2 t}\right\} \subseteq H^{1}\left(P_{m}, \mathbf{Z} / p \mathbf{Z}\right)$ such that $\gamma=\psi_{1} \cup \psi_{2}+\cdots+\psi_{2 t-1} \cup \psi_{2 t}$ and $N \subseteq \bigcap_{i=1}^{t} \operatorname{ker} \psi_{2 i-1}$. Hence, $(\mathbf{Z} / p \mathbf{Z})^{t}$ is a homomorphic image of $P_{m} / N$.

Proof. We argue by induction on $t$. Since $N \cong P_{m}$, we may apply Lemma 3.1 over $N$ to see that $\left\{\operatorname{res}_{P_{m} \rightarrow N}\left(\chi_{i}\right) \mid 1 \leqslant i \leqslant 2 t\right\}$ must be linearly dependent in $H^{1}(N, \mathbf{Z} / p \mathbf{Z})$. That is, for some nonzero linear combination $\delta=a_{1} \chi_{1}$ $+\cdots+a_{2 t} \chi_{2 t} \in H^{1}\left(P_{m}, \mathbf{Z} / p \mathbf{Z}\right), 0=\sum a_{i} \operatorname{res}_{P_{m} \rightarrow N}\left(\chi_{i}\right)=\operatorname{res}_{P_{m} \rightarrow N}(\delta)$. Hence, $N$ $\subseteq \operatorname{ker}(\delta)$. After renumbering the $\chi_{i}$ if necessary and replacing $\delta$ by $a_{1}^{-1} \delta$ we may 
assume $a_{1}=1$; that is, $\chi_{1}=\delta-a_{2} \chi_{2}-\cdots-a_{2 t} \chi_{2 t}$. If $t=1$, we have $\gamma=$ $\chi_{1} \cup \chi_{2}=\delta \cup \chi_{2}$ (as $\chi_{2} \cup \chi_{2}=0^{3}$ ), and the desired result follows by setting $\psi_{1}=\delta$ and $\psi_{2}=\chi_{2}$. Now assume $t>1$. From the formula for $\chi_{1}$ (together with $\chi_{2} \cup \chi_{2}=0^{3}$ ) we find

$$
\begin{aligned}
\gamma= & \delta \cup \chi_{2}+\left(\chi_{3}+a_{4} \chi_{2}\right) \cup\left(\chi_{4}-a_{3} \chi_{2}\right) \\
& +\cdots+\left(\chi_{2 t-1}+a_{2 t} \chi_{2}\right) \cup\left(\chi_{2 t}-a_{2 t-1} \chi_{2}\right) \\
= & \delta \cup \chi_{2}+\sum_{i=2}^{t} \varphi_{2 i-1} \cup \varphi_{2 i},
\end{aligned}
$$

where $\varphi_{2 i-1}=\chi_{2 i-1}+a_{2 i} \chi_{2}$ and $\varphi_{2 i}=\chi_{2 i}-a_{2 i-1} \chi_{2}$. Clearly $\left\{\delta, \chi_{2}, \varphi_{3}, \ldots, \varphi_{2 t}\right\}$ is a base of $\operatorname{span}\left\{\chi_{1}, \ldots, \chi_{2 t}\right\}$.

Let $\gamma^{\prime}=\gamma-\left(\delta \cup \chi_{2}\right)=\sum_{i=2}^{t} \varphi_{2 i-1} \cup \varphi_{2 i}$. Since $N \subseteq \operatorname{ker} \delta$, $\operatorname{res}_{P_{m} \rightarrow N}\left(\gamma^{\prime}\right)=$ $\operatorname{res}_{P_{m} \rightarrow N}(\gamma)=0$. By induction there are $\psi_{3}, \psi_{4}, \ldots, \psi_{2 t} \in H^{1}\left(P_{m}, \mathbf{Z} / p \mathbf{Z}\right)$ with $\operatorname{span}\left\{\psi_{3}, \ldots, \psi_{2 t}\right\}=\operatorname{span}\left\{\varphi_{3}, \ldots, \varphi_{2 t}\right\}, \quad \gamma^{\prime}=\sum_{i=2}^{t} \psi_{2 i-1} \cup \psi_{2 i}, \quad$ and $N \subseteq$ $\bigcap_{i=2}^{t} \operatorname{ker} \psi_{2 i-1}$. Set $\psi_{1}=\delta$ and $\psi_{2}=\chi_{2}$. Clearly $\psi_{1}, \ldots, \psi_{2 t}$ have all the required properties. I.et $K=\bigcap_{i=1}^{t} \operatorname{ker} \psi_{2 i-1} \supseteq N$. Then $P_{m} / K$ is a homomorphic image of $P_{m} / N$, and $P_{m} / K \cong(\mathbf{Z} / p \mathbf{Z})^{t}$ as $\psi_{1}, \psi_{3}, \ldots, \psi_{2 t-1}$ are linearly independent.

4. Free products of pro-p-groups. In this section we prove that, for certain $p$-Henselian fields $L_{1}$ and $L_{2}, G_{p}\left(L_{1} \cap L_{2}\right)$ is the free product of $G_{p}\left(L_{1}\right)$ and $G_{p}\left(L_{2}\right)$ (Theorem 4.3). This will allow us to prove local global principles (Theorem 4.11) relating central simple $\left(L_{1} \cap L_{2}\right)$-algebras to algebras over $L_{1}$ and $L_{2}$. Our main tools are a cohomological characterization of free products of pro-p-groups (Theorem 4.1) and the analogue for pro-p-groups of Kurosch's theorem on subgroups of free products (Theorem 4.5).

Let $\mathscr{G}_{p}$ denote the category of pro-p-groups, a subcategory of the category $\mathscr{G}$ of all groups. For $G_{1}, G_{2} \in \mathscr{G}_{p}$, let $G_{1} *_{p} G_{2}$ denote the free product (coproduct) of $G_{1}$ and $G_{2}$ in $\mathscr{G}_{p}$. (This is not the same as the free product of $G_{1}$ and $G_{2}$ in $\mathscr{G}$, nor even in the category of all profinite groups.) The existence of free products in $\mathscr{G}_{p}$ can be verified by observing that the inclusion functor $\mathscr{G}_{p} \hookrightarrow \mathscr{G}$ has a left adjoint, and free products exist in $\mathscr{G}$. More explicitly, one can construct the free product in $\mathscr{G}_{p}$ as follows: Let $G_{1}, G_{2} \in \mathscr{G}_{p}$. Denote by $G_{1} * G_{2}$ the usual free product of $G_{1}$ and $G_{2}$ in $\mathscr{G}$. Then

$$
G_{1} *_{p} G_{2}=\lim _{N}\left(\left(G_{1} * G_{2}\right) / N\right),
$$

as $N$ ranges over all normal subgroups of $G_{1} * G_{2}$ with $\left|G_{1} * G_{2}: N\right|$ a power of $p$. The easy verification that $G_{1} *_{p} G_{2}$ has the desired universal mapping property is omitted.

\footnotetext{
${ }^{3}$ For any $\chi \in H^{1}\left(P_{m}, \mathbf{Z} / p \mathbf{Z}\right), \chi \cup \chi=0$. For, there is a closed subgroup $H$ of $P_{m}$ with $P_{m} / H \cong P_{1}$ and a $\chi^{\prime} \in H^{1}\left(P_{m} / H, \mathbf{Z} / p \mathbf{Z}\right)$ with $\chi=\inf _{P_{m} / H \rightarrow P_{m}}\left(\chi^{\prime}\right)$. Since $P_{m} / H$ is a free pro-p-group, $H^{2}\left(P_{m} / H, \mathbf{Z} / p \mathbf{Z}\right)=0$. Hence, $\chi \cup \chi=\inf _{P_{m} / H \rightarrow P_{m}}\left(\chi^{\prime} \cup \chi^{\prime}\right)=0$.
} 
The basic cohomological properties of free products in $\mathscr{G}_{p}$ are recalled in convenient form in the next two theorems, which are due to Neukirch [N, Sätze 4.3, 4.2]. (For a version of Theorem 4.1, see also [Er, Proposition 2].) Theorem 4.2 is the pro- $p$ analogue to a well-known result for the usual free products of groups (cf. [HS, p. 220]).

Recall that $\mathbf{Z} / p \mathbf{Z}$ is always viewed as a trivial $G$-module for any $G \in \mathscr{G}_{p}$.

TheOREM 4.1 (Neukirch). Suppose $G, G_{1}, \ldots, G_{k}$ are pro-p-groups, and $f_{j}$ : $G_{j} \rightarrow G$ are continuous homomorphisms, $j=1,2, \ldots, k$. Then the induced map $G_{1} *_{p} G_{2} *_{p} \cdots *_{p} G_{k} \rightarrow G$ is an isomorphism iff the map

$$
H^{i}(G, \mathbf{Z} / p \mathbf{Z}) \rightarrow \bigoplus_{j=1}^{k} H^{i}\left(G_{j}, \mathbf{Z} / p \mathbf{Z}\right)
$$

induced by the $f_{j}$ is an isomorphism for $i=1$ and a monomorphism for $i=2$.

In his version of Theorem 4.1 Neukirch assumes that the $G_{j}$ are subgroups of $G$. But his proof works in the situation described here, without initially assuming the $f_{j}$ to be injective.

TheOrem $4.2(\mathrm{NeUKIRCH})$. Suppose $G_{1}, \ldots, G_{k}$ are pro-p-groups, and that $G=$ $G_{1} *_{p} G_{2} *_{p} \cdots *_{p} G_{k}$. Then for any finite discrete $G$-module $M$ and any $i \geqslant 2$ the map

$$
\text { res: } H^{i}(G, M) \rightarrow \bigoplus_{j=1}^{k} H^{i}\left(G_{j}, M\right)
$$

(induced by the inclusions $G_{j} \hookrightarrow G$ ) is an isomorphism. Moreover, if $M$ is a trivial $G$-module, res is an isomorphism for $i=1$, as well.

The crucial observation needed for our examples is that free products of very nice groups can occur as $G_{p}(F)$ for suitable fields $F$. This is the conclusion of the next key theorem. (A special case of this theorem was proved by a different method in $[\mathbf{J}$, Lemma 9'].)

Two valuations $v_{1}$ and $v_{2}$ on a field $F$ are said to be independent if no proper subring of $F$ contains both valuation rings $V_{F, v_{1}}$ and $V_{F, v_{2}}$. Recall that the approximation theorem [E, (11.16); or $\mathbf{B o}_{2}, \S 7$, No. 2, Theorem 1] holds between any two independent valuations $v_{1}$ and $v_{2}$.

THEOREM 4.3. Let $\left(L_{i}, v_{i}\right)$ be $p$-Henselian valued fields with $\mu_{p} \subseteq L_{i}$ and char $\bar{L}_{i} \neq p$, $i=1$, 2. Let $F=L_{1} \cap L_{2}$. Suppose that the valuations $\left.v_{1}\right|_{F}$ and $\left.v_{2}\right|_{F}$ on $F$ are independent and that the maps $\Gamma_{F, v_{1}} / p \Gamma_{F, v_{i}} \rightarrow \Gamma_{L_{1}} / p \Gamma_{L_{i}}$ and $\bar{F}_{v_{1}} \rightarrow \bar{L}_{v_{1}}$ are surjective, $i=1,2$. Then,

$$
G_{p}(F) \cong G_{p}\left(L_{1}\right){ }_{p} G_{p}\left(L_{2}\right)
$$

Proof. For short we denote the unit group $U_{F, v}$, by $U_{i}$, the value group $\Gamma_{F, v}$, by $\Gamma_{i}$, the residue field $\bar{F}_{v_{1}}$ by $\bar{F}_{i}$, and $H_{p}^{j}\left(K, \mu_{p}\right) \cong H_{p}^{j}(K, \mathbf{Z} / p \mathbf{Z})$ by $H^{j}(K)$ for $K=F$ or $L_{1}$ or $L_{2}$. Let $\operatorname{res}_{F \rightarrow L_{i}}: H^{j}(F) \rightarrow H^{j}\left(L_{i}\right)$ denote the map induced by the restriction homomorphism $G_{p}\left(L_{i}\right) \rightarrow G_{p}(F)$. Set $B_{1}=L_{2}^{p} \cap F^{*}$ and $B_{2}=L_{1}^{p} \cap F^{*}$. 
Consider the commutative diagram:

$$
\begin{aligned}
& 0 \rightarrow\left(U_{1} \cap U_{2}\right) /\left(U_{1} \cap U_{2}\right)^{p} \rightarrow \quad F^{*} / F^{* p} \quad \rightarrow \quad \Gamma_{1} / p \Gamma_{1} \oplus \Gamma_{2} / p \Gamma_{2} \quad \rightarrow 0 \\
& \alpha \downarrow \quad \beta \downarrow \quad \gamma \downarrow \\
& 0 \rightarrow \quad U_{L_{1}} / U_{L_{1}}^{p} \oplus U_{L_{2}} / U_{L_{2}}^{p} \rightarrow L_{1}^{*} / L_{1}^{* p} \oplus L_{2}^{*} / L_{2}^{* p} \rightarrow \Gamma_{L_{1}} / p \Gamma_{L_{1}} \oplus \Gamma_{L_{2}} / p \Gamma_{L_{2}} \rightarrow 0
\end{aligned}
$$

The middle map $\beta$ is injective as $\mu_{p} \subseteq F$ and $F=L_{1} \cap L_{2}$; that is, $B_{1} \cap B_{2}=F^{* p}$. Clearly the bottom row of (*) is exact. Since $\left.v_{1}\right|_{F}$ and $\left.v_{2}\right|_{F}$ are independent, the approximation theorem shows $F^{*}$ maps onto $\Gamma_{1} \oplus \Gamma_{2}$; hence, the top row of (*) is exact. Because $\left(L_{i}, v_{i}\right)$ is $p$-Henselian we have $U_{L_{i}} / U_{L_{i}}^{p} \cong \bar{L}_{i}^{*} / \bar{L}_{i}^{* p}$ (see Lemma 1.8(ii)). It follows that $\alpha$ is surjective by the approximation theorem and the hypothesis that $\bar{F}_{i} \cong \bar{L}_{i}$. By hypothesis, $\gamma$ is also surjective. Thus, the snake lemma $\left[\mathbf{B o}_{1}, \S 1\right.$, Proposition 2] implies that $\beta$ and $\gamma$ are isomorphisms. Hence, $B_{i} / F^{* p} \cong$ $L_{i}^{*} / L_{i}^{* p}, i=1,2, B_{1} \cdot B_{2}=F^{*}$, and $p \Gamma_{L_{i}} \cap \Gamma_{i}=p \Gamma_{i}, i=1$, 2. In cohomological terms, the isomorphism $\beta$ reads: $H^{1}(F) \cong H^{1}\left(L_{1}\right) \oplus H^{1}\left(L_{2}\right)$.

Let $C_{i j}$ denote the subgroup of $H^{2}(F)$ generated by the cup products $\{(a) \cup$ (b) $\left.\mid a \in B_{i}, b \in B_{j}\right\}$ for $i=1,2, j=1,2$. Since $B_{1} \cdot B_{2}=F^{*}$ the Merkurjev-Suslin Theorem 1.3 and the remarks after (1.7) show that $H^{2}(F)=C_{11}+C_{12}+C_{22}$. We will prove that $C_{12}=0$ and $H^{2}(F)=C_{11} \oplus C_{22}$, with $C_{i i} \cong H^{2}\left(L_{i}\right)$ via the restriction map. Since clearly res $\operatorname{res}_{F \rightarrow L_{1}}\left(C_{22}\right)=\operatorname{res}_{F \rightarrow L_{2}}\left(C_{11}\right)=0$ it follows that res: $H^{2}(F)$ $\rightarrow H^{2}\left(L_{1}\right) \oplus H^{2}\left(L_{2}\right)$ is an isomorphism. Then the desired conclusion $G_{p}(F) \cong$ $G_{p}\left(L_{1}\right) *_{p} G_{p}\left(L_{2}\right)$ follows by Neukirch's Theorem 4.1 .

Consider any generator $(a) \cup(b)$ of $C_{12}$ with $a \in B_{1}, b \in B_{2}$. As $a \in L P$, using $p \Gamma_{L_{2}} \cap \Gamma_{2}=p \Gamma_{2}$, the surjectivity of $\bar{F}_{2} \rightarrow \bar{L}_{2}$, and the approximation theorem we may find an $a^{\prime} \in F^{*}$ with $a^{\prime} \equiv a \bmod F^{* p}, v_{2}\left(a^{\prime}\right)=0, \overline{a^{\prime}}=1$ in $\bar{F}_{2}$, and $v_{1}\left(a^{\prime}\right)>0$. Likewise, there is a $b^{\prime} \equiv b \bmod F^{* p}$ with $v_{1}\left(b^{\prime}\right)=0, \overline{b^{\prime}}=1$ in $\bar{F}_{1}$, and $v_{2}\left(b^{\prime}\right)>0$. Consequently $v_{i}\left(a^{\prime}+b^{\prime}\right)=0$ and $\overline{a^{\prime}+b^{\prime}}=1$ in $\bar{L}_{i}$ for $i=1$ and $i=2$. Since the $L_{i}$ are $p$-Henselian it follows by Lemma 1.8(i) that $a^{\prime}+b^{\prime} \in L_{1}^{p} \cap L L_{2}^{p} \cap F=F^{p}$. Thus, $\left(a^{\prime}\right) \otimes\left(b^{\prime}\right)$ lies in the Steinberg relation subgroup of $H^{1}(F) \otimes H^{1}(F)$ (cf. Theorem 1.3). From this we see that $(a) \cup(b)=\left(a^{\prime}\right) \cup\left(b^{\prime}\right)=0$ in $H^{2}(F)$. Thus, $C_{12}=0$.

To complete the proof we must show $H^{2}(F)=C_{11} \oplus C_{22} \cong H^{2}\left(L_{1}\right) \oplus H^{2}\left(L_{2}\right)$. This is immediate from the following

Claim. There exist homomorphisms $\varepsilon_{i}: H^{2}\left(L_{i}\right) \rightarrow H^{2}(F)$, with $\operatorname{im}\left(\varepsilon_{i}\right)=C_{i i}$ and $\operatorname{res}_{F \rightarrow L_{i}}{ }^{\circ} \varepsilon_{i}: H^{2}\left(L_{i}\right) \rightarrow H^{2}\left(L_{i}\right)$ the identity map, $i=1,2$.

To prove the claim, observe first that the isomorphism $B_{i} / F^{* p} \cong L_{i}^{*} / L_{i}^{* p}$ yields an injection $\hat{\varepsilon}_{i}: H^{1}\left(L_{i}\right) \rightarrow H^{1}(F)$ which is the composite of

$$
H^{1}\left(L_{i}\right) \stackrel{\cong}{\rightarrow} L_{i}^{*} / L_{i}^{* p} \stackrel{\cong}{\rightarrow} B_{i} / F^{* p} \hookrightarrow F^{*} / F^{* p} \stackrel{\cong}{\rightarrow} H^{1}(F) .
$$

Note that $\hat{\varepsilon}_{i}\left((a)_{L_{i}}\right)=(a)_{F}$ for every $a \in B_{i}$. The map $\hat{\varepsilon}_{i} \otimes \hat{\varepsilon}_{i}$ composed with the cup product yields a homomorphism

$$
\varepsilon_{i}^{\prime}: H^{1}\left(L_{i}\right) \otimes H^{1}\left(L_{i}\right) \rightarrow H^{2}(F)
$$


defined on generators by $\varepsilon_{i}^{\prime}\left((a)_{L_{,}} \otimes(b)_{L_{i}}\right)=(a)_{F} \cup(b)_{F}$ for all $a, b \in B_{i}$. Since $\operatorname{im}\left(\varepsilon_{i}^{\prime}\right)=C_{i i}$ and the composition $\operatorname{res}_{F \rightarrow L_{i}} \circ \hat{\varepsilon}_{i}: H^{1}\left(L_{i}\right) \rightarrow H^{1}\left(L_{i}\right)$ is identity, to prove the claim it suffices to show that $\varepsilon_{i}^{\prime}$ induces a well-defined homomorphism $\varepsilon_{i}$ : $H^{2}\left(L_{i}\right) \rightarrow H^{2}(F)$. In view of the Merkurjev-Suslin Theorem 1.3 we must show that $S_{i} \subseteq$ ker $\varepsilon_{i}^{\prime}$, where $S_{i}$ is the Steinberg relation subgroup of $H^{1}\left(L_{i}\right) \otimes H^{1}\left(L_{i}\right)$. That is, Steinberg relations can be "lifted" from $L_{i}$ to $F$.

Take any generator $(a)_{L_{i}} \otimes(b)_{L_{-}}$of $S_{i}, a, b \in B_{i}$. Then there exist $r, s \in L_{i}^{*}$ such that

$$
a r^{p}+b s^{p}=1 .
$$

In showing $(a)_{F} \cup(b)_{F}=0$ there are four cases to consider:

First, suppose $v_{i}\left(a r^{p}\right) \neq 0$ and $v_{i}\left(b s^{p}\right) \neq 0$. Then valuation theory and equation (†) show that $v_{i}\left(a r^{p}\right)=v_{i}\left(b s^{p}\right)<0$ and $-a r^{p}\left(b s^{p}\right)^{-1} \in 1+M_{L_{i}}$. This yields $-a b^{-1}$ $\in L_{i}^{p}$ since $L_{i}$ is $p$-Henselian. As $L_{i}^{p} \cap B_{i}=F^{* p}$ we find $-a b^{-1} \in F^{* p}$, i.e., $(b)_{F}=(-a)_{F}$ in $H^{1}(F)$. So $\varepsilon_{l}^{\prime}\left((a)_{L_{l}} \otimes(b)_{L_{l}}\right)=(a)_{F} \cup(b)_{F}=(a)_{F} \cup(-a)_{F}=0$ in $H^{2}(F)$ (cf. [Mi, p. 319]).

For the next case suppose $v_{i}\left(a r^{p}\right)=0$ but $v_{i}\left(b s^{p}\right) \neq 0$. Then from ( $\dagger$ ) we have $v_{i}\left(b s^{p}\right)>0$ and $a r^{p} \in 1+M_{L_{i}} \subseteq L_{i}^{p}$. Thus, $a \in L_{i}^{p} \cap B_{i}=F^{* p}$. So, $(a)_{F}=0$ in $H^{1}(F)$, which assures $(a)_{F} \cup(b)_{F}=0$ in $H^{2}(F)$. The case where $v_{i}\left(a r^{p}\right) \neq 0$ but $v_{i}\left(b s^{p}\right)=0$ is handled analogously.

In the final case, we have $v_{i}\left(a r^{p}\right)=v_{i}\left(b s^{p}\right)=0$. So, $v_{i}(a), v_{i}(b) \in p \Gamma_{i}$ by the injectivity of the map $\gamma$ of diagram (*). Modifying $a$ and $b$ by $p$ th powers from $F$, we may assume that each of $a, b, r, s$ lies in $U_{L_{i}}$. Recall that $a \in B_{i} \subseteq L_{j}^{p}$, where $j=3-i$. So $v_{j}(a) \in p \Gamma_{L_{j}} \cap \Gamma_{j}=p \Gamma_{j}$. Applying the approximation theorem and the isomorphism $\bar{F}_{i} \cong \bar{L}_{i}$, we can choose $r^{\prime}, s^{\prime} \in F^{*}$ with $v_{i}\left(r^{\prime}\right)=0, \overline{r^{\prime}}=\bar{r}$ in $\bar{L}_{i}$, $v_{j}\left(r^{\prime p}\right)=-v_{j}(a)$, and $v_{i}\left(s^{\prime}\right)=0, \overline{s^{\prime}}=\bar{s}$ in $\bar{L}_{i}, v_{j}\left(s^{\prime p}\right)>-v_{j}(b)$. Then $a r^{p}+b s^{\prime p}$ is a unit with respect to each valuation, and $\overline{a r^{\prime p}+b s^{\prime p}}=\overline{a r^{p}+b s^{p}}=1$ in $\bar{L}_{i}$ and $\overline{a r^{\prime p}+b s^{\prime p}}=\overline{a r^{\prime p}}$ in $\bar{L}_{j}^{p}$. Since $L_{1}$ and $L_{2}$ are each $p$-Henselian, it follows that $a r^{p}+b s^{\prime p} \in L_{1}^{p} \cap L_{2}^{p} \cap F=F^{p}$. Hence, $(a)_{F} \otimes(b)_{F}$ lies in the Steinberg relation group of $H^{1}(F) \otimes H^{1}(F)$. Thus, $(a)_{F} \cup(b)_{F}=0$ in $H^{2}(F)$. This establishes the claim and completes the proof of Theorem 4.3.

Remark 4.4. Suppose $\left(L_{i}, v_{i}\right)$ are $p$-Henselian valued fields, $i=1$, 2, with $\mu_{p} \subseteq L_{i}$ and char $\bar{L}_{i} \neq p$ for each $i$. Let $F=L_{1} \cap L_{2}$, and suppose $\left.v_{1}\right|_{F}$ and $\left.v_{2}\right|_{F}$ are independent valuations. In each of the following two situations we can see that each $\left(L_{i}, v_{i}\right)$ is an immediate extension of $\left(F, v_{i}\right)$, so the hypotheses of Theorem 4.3 are satisfied:

(i) Each $L_{i}$ is unramified over $F, \bar{L}_{i}$ is Galois over $\bar{F}_{v_{i}}$ and $\left(L_{i}, v_{i}\right)$ is Henselian.

(ii) Each $L_{i}$ is unramified over $F$ and $L_{i} \subseteq \tilde{F}_{p}$, the $p$ th root closure of $F$.

In either case, it suffices to check that $\bar{F}_{i}=\bar{F}_{v_{i}}$ maps onto $\bar{L}_{i}$. In case (i) take any $\bar{c} \in \bar{L}_{i}$, and let $\bar{f}_{i} \in \bar{F}_{i}[X]$ be the minimal polynomial of $\bar{c}$ over $\bar{F}_{i}$. Then $\bar{f}_{i}$ splits completely over $\bar{L}_{i}$, as $\bar{L}_{i}$ is Galois over $\bar{F}_{i}$. For $j=3-i$, pick any monic $\bar{f}_{j} \in \bar{F}_{j}[X]$ with $\operatorname{deg} \bar{f}_{j}=\operatorname{deg} \bar{f}_{i}$, and such that $\bar{f}_{j}$ splits completely in $\bar{F}_{j}[X]$ with no repeated roots. By the approximation theorem applied to the corresponding coefficients of $\bar{f}_{i}$ and $\bar{f}_{j}$ there is a monic $f \in V_{F, v_{1}}[X] \cap V_{F, v_{2}}[X]$ with $\bar{f}=\bar{f}_{1}$ in $\bar{F}_{1}[X]$ 
and $\bar{f}=\bar{f}_{2}$ in $\bar{F}_{2}[X]$. The Henselian assumption implies that $f$ splits in $L_{1}$ and $L_{2}$. Hence, $f$ splits in $L_{1} \cap L_{2}=F$. So, $\bar{c} \in \bar{F}_{i}$ which shows that $\bar{L}_{i}=\bar{F}_{i}$. In case (ii) we have $\mu_{p} \subseteq \bar{F}_{i}$ and $\bar{L}_{i} \subseteq \tilde{\bar{F}}_{p}$. From the theory of $p$-groups (cf. (1.4)) if $\bar{F}_{i} \neq \bar{L}_{i}$, then there is a $\bar{d} \in \bar{L}_{i}-\bar{F}_{i}$ with $\bar{d}^{p} \in \bar{F}_{i}$. Pick any $\bar{e} \in \bar{F}_{j}^{*}, j=3-i$. By the approximation theorem there is a $b \in F^{*}$ with $v_{1}(b)=v_{2}(b)=0$ and $\bar{b}=\bar{d}^{p}$ in $\bar{F}_{i}$ and $\bar{b}=\bar{e}^{p}$ in $\bar{F}_{j}$. Since $L_{1}$ and $L_{2}$ are $p$-Henselian, we have $b \in L_{1}^{p} \cap L_{2}^{p} \cap F=F^{p}$. Hence $\bar{d}^{p}=\bar{b} \in \bar{F}_{i}^{p}$, contradicting the choice of $\bar{d}$.

We will exploit Theorem 4.3 below to obtain local global principles relating algebras over $F$ to their extensions over $L_{1}$ and $L_{2}$. The key to moving from cohomological data to information about algebras is provided by some index computations which are consequences of the pro- $p$ version of the famous Kurosch subgroup theorem. This theorem is due to Binz, Neukirch, and Wenzel [BNW]-in a more general form than given here. In what follows, we write $H^{g}$ for the conjugate $g \mathrm{Hg}^{-1}$ of a group $H$.

Theorem 4.5 (Binz, Neukirch, Wenzel). Suppose $G, G_{1}, \ldots, G_{k}$ are pro-p-groups with $G=G_{1}{ }_{p} G_{2} *_{p} \cdots *_{p} G_{k}$. Let $H$ be an open subgroup of $G$. Then,

$$
H={ }_{i=1}^{*}\left({ }_{j=1}^{n_{p}} G_{i}^{g_{i j}} \cap H\right) *_{p} \mathscr{F},
$$

where for each $i$ the $g_{i 1}, \ldots, g_{i n}$ are a full set of representatives for the double cosets $H g G_{i}$ of $H$ and $G_{i}$ in $G$, and $\mathscr{F}$ is a free pro-p-group.

Before turning to central simple algebras we consider the notion of index in a purely cohomological setting:

Definition 4.6. Let $G$ be a pro-p-group and let $\gamma \in H^{i}(G, M), i \geqslant 2$, for some discrete $G$-module $M$. The $p$-index of $\gamma$ is

$$
\begin{aligned}
& p \text {-ind }(\gamma):=\min \{|G: H| \mid H \text { is an open subgroup of } G \text { and } \\
& \left.\qquad \operatorname{res}_{G \rightarrow H}(\gamma)=0 \text { in } H^{i}(H, M)\right\} .
\end{aligned}
$$

REMARKS 4.7. (i) $p$-ind $(\gamma)$ is always finite. For, as $M$ is discrete and $\gamma$ is a continuous cohomology class, there is an open normal subgroup $N$ of $G$ with

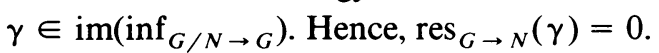

(ii) If $K$ is a closed subgroup of $G$, then $p$-ind $\left(\operatorname{res}_{G \rightarrow K}(\gamma)\right) \leqslant p$-ind $(\gamma)$.

(iii) If $\operatorname{res}_{G \rightarrow H}(\gamma)=0$, we say that $H$ splits $\gamma$. Note that if $H$ splits $\gamma$, then every conjugate $H^{g}$ of $H$ in $G$ also splits $\gamma$. For, the conjugation map $H \rightarrow H^{g}$ induces a function $c_{g, H}: H^{i}(H, M) \rightarrow H^{i}\left(H^{g}, M\right)$. Since $c_{g, G}$ is the identity map on $H^{i}(G, M)$ (cf. [Se $\mathbf{S}_{2}$, p. 116, Proposition 3; or We, p. 65, Proposition 2-3-1]), we have

$$
\operatorname{res}_{G \rightarrow H^{g}}(\gamma)=\left(\operatorname{res}_{G \rightarrow H^{g}} \circ c_{g, G}\right)(\gamma)=\left(c_{g, H} \circ \operatorname{res}_{G \rightarrow H}\right)(\gamma)=0 .
$$

THEOREM 4.8. Suppose $H_{1}, \ldots, H_{k}$ are closed subgroups of a pro-p-group $G$, and suppose $G=H_{1} *_{p} \cdots *_{p} H_{k}$. Then, for any discrete $G$-module $M$ and any $\gamma \in$ $H^{i}(G, M), i \geqslant 2$,

$$
p \text {-ind }(\gamma)=\max _{1 \leqslant j \leqslant k}\left\{p \text {-ind }\left(\operatorname{res}_{G \rightarrow H_{j}}(\gamma)\right)\right\}
$$


Proof. Let $p^{m}=\max _{1 \leqslant j \leqslant k}\left\{p\right.$-ind( $\left.\left.\operatorname{res}_{G \rightarrow H}(\gamma)\right)\right\}$. By Remark 4.7(ii), $p$-ind $(\gamma) \geqslant$ $p^{m}$. To prove the reverse inequality we proceed by induction on $m$. If $m=0$, each $H_{i}$ splits $\gamma$, so as $H^{i}(G, M) \cong \oplus_{j=1}^{k} H^{i}\left(H_{j}, M\right)$ by Theorem 4.2 , we find that $G$ splits $\gamma$, i.e., $p$-ind $(\gamma)=1=p^{0}$.

Now, suppose $m \geqslant 1$. For each $j$, choose an open subgroup $K_{j}$ of $H_{j}$ such that $K_{j}$ splits $\gamma$ and $\left|H_{j}: K_{j}\right|=p$-ind(res $\left.\operatorname{raH}_{j}(\gamma)\right)$. If $K_{j} \neq H_{j}$, let $N_{j}$ be a maximal proper subgroup of $H_{j}$ containing $K_{j}$; so $N_{j}$ is normal in $H_{j}$ and $H_{j} / N_{j} \cong \mathbf{Z} / p \mathbf{Z}$, as $H_{j}$ is a pro-p-group. If $K_{j}=H_{j}$, let $N_{j}=H_{j}$. Then for each $j$ there is a homomorphism $\pi_{j}$ : $H_{j} \rightarrow \mathbf{Z} / p \mathbf{Z}$ with kernel $N_{j}$. At least one $\pi_{j}$ is surjective, as $m \geqslant 1$. By the universal mapping property of the free product there is an epimorphism $\pi: G \rightarrow \mathbf{Z} / p \mathbf{Z}$ with $\left.\pi\right|_{H_{1}}=\pi_{j}$ for each $j$. Set $N=\operatorname{ker}(\pi)$. Then $N$ is a normal subgroup of $G$, $|G: N|=p$, and $N \cap H_{j}=N_{j} \supseteq K_{j}$ for $j=1,2, \ldots, k$.

Applying Theorem 4.5 we have

$$
N=L_{1} *_{p} \cdots *{ }_{p} L_{l} *_{p} \mathscr{F}
$$

where $\mathscr{F}$ is a free pro-p-group and each $L_{i}=N \cap H_{j(i)} g_{i}=\left(N \cap H_{j(i)}\right)^{g_{\imath}}$ for some $g_{i} \in G$ and $j(i) \in\{1,2, \ldots, k\}$. Set $K_{i}^{\prime}=K_{j(i)} g_{i} \subseteq L_{i}$ and set $\delta=\operatorname{res}_{G \rightarrow N}(\gamma)$. Since $K_{j(i)}$ splits $\gamma, K_{i}^{\prime}$ must also split $\gamma$ (hence $\delta$ ), by Remark 4.7(iii). Consequently,

$$
p \text {-ind }\left(\operatorname{res}_{N \rightarrow L_{i}}(\delta)\right) \leqslant\left|L_{i}: K_{i}^{\prime}\right|=\left|N_{j(i)}: K_{j(i)}\right| \leqslant p^{m-1} .
$$

Also, $p$-ind $\left(\operatorname{res}_{N \rightarrow \mathscr{F}}(\delta)\right)=p^{0}$ as $H^{i}(\mathscr{F}, M)=0, \quad i \geqslant 2$. Thus, by induction, $p$-ind $(\delta) \leqslant p^{m-1}$, so that

$$
p \text {-ind }(\gamma) \leqslant|G: N| \cdot p \text {-ind }\left(\operatorname{res}_{G \rightarrow N}(\gamma)\right) \leqslant p \cdot p^{m-1}=p^{m} .
$$

This proves the theorem.

Definition 4.9. Let $A$ be a central simple $F$-algebra with $[A] \in \operatorname{Br}_{p}(F)$. The p-index of $A$ is

$$
p \text {-ind }(A):=\min \left\{[L: F] \mid L \text { is a field, } F \subseteq L \subseteq \tilde{F}_{p} \text {, and } L \text { splits } A\right\} .
$$

Remarks 4.10. (i) For every $A$ with $[A] \in \operatorname{Br}_{p}(F), p$-ind $(A)$ is necessarily finite. For, as we observed in $\S 1$, every such $A$ is split by $\tilde{F}_{p}$, so by some finite degree subextension.

(ii) If $\mu_{p} \subseteq F$, then $G_{p}(F)$ is a pro-p-group. If [ $A$ ] has exponent $p^{n}$ there is a corresponding element $\gamma$ of $H_{p}^{2}\left(F, \mu_{p^{n}}\right)$. Then for any field $M \supseteq F$, $\operatorname{res}_{F \rightarrow M}(\gamma)$ is the element of $H_{p}^{2}\left(M, \mu_{p^{n}}\right)$ corresponding to $\left[M \otimes_{F} A\right]$ in $\operatorname{Br}_{p}(M)$. From this it is clear that $p$-ind $(A)=p$-ind $(\gamma)$, which is a power of $p$. However, if $\mu_{p} \notin F$, it is unknown whether $p$-ind $(A)$ is always a $p$-power.

(iii) For any $A$ with $[A] \in \operatorname{Br}_{p}(F)$, clearly index $(A) \leqslant p$-ind $(A)$, but it is an open question whether equality always holds. (Equality means that the underlying division algebra of $A$ has a maximal subfield in $\tilde{F}_{p}$.) Indeed, if $\mu_{p} \subseteq F$ and $\operatorname{index}(A)=p$, then $p$-ind $(A)=p$ iff the underlying division algebra of $A$ is a cyclic algebra. But for $p \geqslant 5$ ( $p$ prime) it is unknown whether every division algebra of index $p$ is cyclic. 
Theorem 4.11 (Local-Global Principles). Let $L_{1}$ and $L_{2}$ be fields with $\mu_{p} \subseteq L_{i}, i=1,2$, and let $F=L_{1} \cap L_{2}$. Suppose the natural map $G_{p}\left(L_{1}\right) *_{p} G_{p}\left(L_{2}\right)$ $\rightarrow G_{p}(F)$ is an isomorphism. Then for any central simple $F$-algebra $A$ with $[A] \in$ $\operatorname{Br}_{p}(F)$,

(i) $F$ splits $A$ iff $L_{1}$ and $L_{2}$ each split $A$;

(ii) $\operatorname{index}(A) \leqslant p$-ind $(A)=\max \left\{p\right.$-ind $\left.\left(A \otimes_{F} L_{i}\right) \mid i=1,2\right\}$;

(iii) if $\operatorname{index}\left(A \otimes_{F} L_{i}\right)=p$-ind $\left(A \otimes_{F} L_{i}\right), i=1,2$, then $\operatorname{index}(A)=p$-ind $(A)$;

(iv) for any field $K$ with $F \subseteq K \subseteq \tilde{F}_{p}$ and $[K: F]<\infty, K$ splits $A$ iff $K \otimes_{F} L_{i}$ splits $A, i=1,2$;

(v) suppose there are finite degree Galois p-extensions $M_{i}$ of $L_{i}$ which split $A$, and suppose $G$ is a finite p-group generated by isomorphic copies of $\mathscr{G}\left(M_{i} / L_{i}\right), i=1,2$; then there is a Galois extension $M$ of $F$ for which $\mathscr{G}(M / F) \cong G, M$ splits $A$ and $M \cdot L_{i}=M_{i}, i=1,2$.

Note that for $K$ as in (iv), $K$ is separable over $F$, so $K \otimes_{F} L_{i}$ is a direct sum of fields. " $K \otimes_{F} L_{i}$ splits $A$ " means each summand splits $A$.

Proof. Suppose [A] has exponent $p^{n}$; let $\gamma \in H_{p}^{2}\left(F, \mu_{p^{n}}\right)$ be the element corresponding to $[A]$ in ${ }_{p^{n}} \operatorname{Br}(F)$.

Part (i) is immediate from the isomorphism $H_{p}^{2}\left(F, \mu_{p^{n}}\right) \cong H_{p}^{2}\left(L_{1}, \mu_{p^{n}}\right) \oplus$ $H_{p}^{2}\left(L_{2}, \mu_{p^{n}}\right)$ given by Theorem 4.2. Part (ii) follows from Theorem 4.8 and Remarks 4.10(ii), (iii). Since $\operatorname{index}(A) \geqslant \max \left\{\operatorname{index}\left(A \otimes_{F} L_{i}\right) \mid i=1,2\right\}$, (iii) is immediate from (ii).

For (iv) the "only if" part is clear. For the reverse implication assume that (each summand of) each $K \otimes_{F} L_{i}$ splits $A$. Let $N=G_{p}(K)$, an open subgroup of $G_{p}(F)$. Let $H_{i}=G_{p}\left(L_{i}\right)$, which we identify with its image in $G_{p}(F)$. (The free product hypothesis assures that the map $H_{i} \rightarrow G_{p}(F)$ is injective.) Let

$$
N=N_{11} *_{p} \cdots *_{p} N_{1 n_{1}} *_{p} N_{21} *_{p} \cdots *_{p} N_{2 n_{2}} *_{p} \mathscr{F}
$$

be the free product decomposition of $N$ given by Theorem 4.5, where $N_{i j}=H_{i}{ }^{g_{i j}} \cap N$ for suitable $g_{i j} \in G_{p}(F)$ and $\mathscr{F}$ is a free pro-p-group. For each $i, j, H_{i} \cap N^{g_{i j}^{-1}}$ is (isomorphic to) $G_{p}\left(g_{i j}^{-1}(K) \cdot L_{i}\right)$. Since the compositum $g_{i j}^{-1}(K) \cdot L_{i}$ is isomorphic to a summand of $K \otimes_{F} L_{i}$, it splits $A$. Thus, $\operatorname{res}_{G \rightarrow\left(H_{i} \cap N_{i j}^{g-1}\right)}(\gamma)=0$. Let $\delta=$ $\operatorname{res}_{G \rightarrow N}(\gamma)$. Since $H_{i} \cap N^{g_{i j}^{-1}}=N_{i j}^{g_{i j}^{-1}}$, we have $0=\operatorname{res}_{G \rightarrow N_{i j}}(\gamma)=\operatorname{res}_{N \rightarrow N_{i j}}(\delta)$ (cf. Remark 4.7(iii)). Because $\mathscr{F}$ is free, $\operatorname{res}_{N \rightarrow \mathscr{F}}(\delta)=0$. Therefore, by Theorem 4.2 $\delta=0$ in $H^{2}\left(N, \mu_{p^{n}}\right)$, i.e., $K$ splits $A$, as desired.

(v) Let $f_{i}$ be the composite homomorphism $G_{p}\left(L_{i}\right) \rightarrow \mathscr{G}\left(M_{i} / L_{i}\right) \hookrightarrow G$, with kernel $G_{p}\left(M_{i}\right)$; we have an induced epimorphism $f: G_{p}(F) \stackrel{\cong}{\rightarrow} G_{p}\left(L_{1}\right) *_{p} G_{p}\left(L_{2}\right) \rightarrow$ $G$. Let $M$ be the fixed field of $\operatorname{ker} f$. Then $M$ is Galois over $F$ and $\mathscr{G}(M / F) \cong$ $G_{p}(F) / \operatorname{ker} f \cong G$. When we identify $G_{p}(F)$ with $G_{p}\left(L_{1}\right) *_{p} G_{p}\left(L_{2}\right), \operatorname{ker} f \cap G_{p}\left(L_{i}\right)$ $=\operatorname{ker} f_{i}=G_{p}\left(M_{i}\right)$. Consequently, $M \cdot L_{i}=M_{i}$. Since $M$ is Galois over $F$ every summand of $M \otimes_{F} L_{i}$ is isomorphic to $M \cdot L_{i}$. Hence, $M \otimes_{F} L_{i}$ splits $A$. By (iv) $M$ splits $A$, as desired.

REMARK. Theorems 4.3 and 4.11 which were stated for $F=L_{1} \cap L_{2}$ clearly hold as well for $F=L_{1} \cap L_{2} \cap \cdots \cap L_{k}$ for any integer $k \geqslant 2$. 
5. Noncrossed products of exponent $p^{n}, n \geqslant 3$. Fix a prime number $p$ and integers $m \geqslant n \geqslant 3$. In this section we will construct a noncrossed product division algebra $D$ of exponent $p^{n}$ and index $p^{m}$ over a field $F$ of any characteristic $\neq p$.

To begin, fix any field $k$ containing $p$ distinct $p$ th roots of unity (so char $k \neq p$ ). Set $F_{0}=k\left(x_{1}, x_{2}, y_{1}, y_{2}, y_{3}, \ldots, y_{2 m}\right)$, where $x_{1}, x_{2}, y_{1}, \ldots, y_{2 m}$ are algebraically independent over $k$. Let $v_{1}$ be the valuation on $F_{0}$ with residue field $k\left(y_{1}, \ldots, y_{2 m}\right)$ and value group $\mathbf{Z} \times \mathbf{Z}$, ordered lexicographically, with $v_{1}\left(x_{1}\right)=(1,0)$ and $v_{1}\left(x_{2}\right)$ $=(0,1)$. (This is the valuation described in Example 2.7, viewing $F_{0}=K\left(x_{1}, x_{2}\right)$, with $K=k\left(y_{1}, \ldots, y_{2 m}\right)$.) Let $v_{2}$ be the valuation on $F_{0}$ with residue field $k\left(x_{1}, x_{2}\right)$ and value group $\prod_{i=1}^{2 m} \mathbf{Z}$, ordered lexicographically, with $v_{2}\left(y_{i}\right)=(0, \ldots, 0,1,0, \ldots, 0)$ (the 1 in the $i$ th place). (This is another case of Example 2.7, viewing $F_{0}=$ $K\left(y_{1}, \ldots, y_{2 m}\right)$ with $K=k\left(x_{1}, x_{2}\right)$.) Clearly $v_{1}$ and $v_{2}$ are independent valuations on $F_{0}$. Within some fixed algebraic closure of $F_{0}$ let $\left(L_{1}, v_{1}\right)$ be a strict $p$-Henselization of $\left(F_{0}, v_{1}\right)$ and let $\left(L_{2}, v_{2}\right)$ be a strict $p$-Henselization of $\left(F_{0}, v_{2}\right)$, as described in $\S 1$.

Let $F=L_{1} \cap L_{2}$. Note that $F$ contains a primitive $p^{n}$ th root of unity $\omega$ as $\mu_{p^{\prime}} \subseteq L_{i}$ for all $l, i=1$, 2. Let $\rho=\omega^{p^{n-1}}$, a primitive $p$ th root of unity. Using the notation of $\S 1$ we define central simple $F$-algebras $A_{1}$ and $A_{2}$ by

$$
A_{1}=A_{\omega}\left(x_{1}, x_{2} ; F\right) \text { and } A_{2}=\bigotimes_{j=1}^{m} A_{\rho}\left(y_{2 j-1}, y_{2 j} ; F\right) \text {. }
$$

Set $A=A_{1} \otimes_{F} A_{2}$. By Wedderburn's theorem $A \cong M_{l}(D)$, where $D$ is a division algebra with center $F$. The notation defined here will remain fixed throughout this section. We will show in Theorem 5.4 that $D$ is not a crossed product. But first we summarize the nice properties of $F$ given by our earlier theorems.

THEOREM 5.1. With $F, L_{1}, L_{2}$, and $p$ as above, we have

(i) $G_{p}\left(L_{1}\right) \cong \hat{\mathbf{Z}}_{p} \oplus \hat{\mathbf{Z}}_{p}$ and $G_{p}\left(L_{2}\right) \cong\left(\hat{\mathbf{Z}}_{p}\right)^{m}$;

(ii) $G_{p}(F) \cong G_{p}\left(L_{1}\right) *_{p} G_{p}\left(L_{2}\right)$;

(iii) $\mathrm{Br}_{p}(F) \cong \mathrm{Br}_{p}\left(L_{1}\right) \oplus \mathrm{Br}_{p}\left(L_{2}\right)$;

(iv) the local global principles (Theorem 4.11) apply from $L_{1}$ and $L_{2}$ to $F$.

Proof. Since the value group $\Gamma_{L_{r}}=\Gamma_{F_{0}, v_{i}}$, (i) follows from Lemma 1.9(iv). The valuations $v_{1}$ and $v_{2}$ are independent on $F$ since they are independent on $F_{0}$ and $F$ is algebraic over $F_{0}$. Thus, (ii) follows from Remark 4.4(ii) and Theorem 4.3. Then (iii) follows by Theorem 4.2, taking $M=\mu_{p^{\prime}}, l=1,2, \ldots$ Finally, (ii) implies (iv).

ReMARK 5.2. It can be shown (though we will not) that over a strictly $p$-Henselian field $L$ every central simple division algebra $B$ with $[B] \in \operatorname{Br}_{p}(L)$ is isomorphic to a tensor product of cyclic algebras. Hence, $\operatorname{index}(B)=p$-ind $(B)$. Using this and Theorem 4.11(iii) we can add the following properties of $F$ to the list in 5.1: Let $C$ be any central simple $F$-algebra with $[C] \in \mathrm{Br}_{p}(F)$; then

(v) index $(C)=p$-ind $(C)=\max \left\{p\right.$-ind $\left.\left(C \otimes_{F} L_{i}\right) \mid i=1,2\right\}$;

(vi) if $C$ is a division algebra, then $C \otimes_{F} L_{1}$ is a division algebra or $C \otimes_{F} L_{2}$ is a division algebra. 
Lemma 5.3. (i) $A_{1} \otimes_{F} L_{1}$ is a division algebra of exponent, index, and p-index $p^{n}$, while $L_{2}$ splits $A_{1}$.

(ii) $A_{2} \otimes_{F} L_{2}$ is a division algebra of index and p-index $p^{m}$, while $L_{1}$ splits $A_{2}$. If $M$ is a Galois extension of $L_{2}$ which splits $A_{2}$ and $\left[M: L_{2}\right]$ is a power of $p$, then $(\mathbf{Z} / p \mathbf{Z})^{m}$ is a homomorphic image of $\mathscr{G}\left(M / L_{2}\right)$.

Proof. (i) Note that $A_{1} \otimes_{F} L_{1} \cong A_{\omega}\left(x_{1}, x_{2} ; L_{1}\right) \cong A_{1}^{\prime} \otimes_{F_{0}} L_{1}$, where $A_{1}^{\prime}=$ $A_{\omega}\left(x_{1}, x_{2} ; F_{0}\right)$. Example 2.7 with the valuation $v_{1}$ on $F_{0}$ shows that $A_{1}^{\prime}$ is a division algebra, and that $A_{1}^{\prime} \otimes_{F_{0}} L_{1}$ is also a division algebra, as $\left(L_{1}, v_{1}\right)$ is unramified over $\left(F_{0}, v_{1}\right)$. Since $A_{1} \otimes_{F} L_{1}$ is a crossed product division algebra, $p$-ind $\left(A \otimes_{F} L_{1}\right)=$ index $\left(A \otimes_{F} L_{1}\right)=p^{n}$. The valuation $v_{1}$ on $L_{1}$ extends uniquely to $L_{1}^{\prime}:=L_{1}\left(x_{1}^{1 / p^{n}}\right)$ with value group $p^{-n} \mathbf{Z} \times \mathbf{Z}$. So, $v_{1}$ maps the norm group $N_{L_{1}^{\prime} / L_{1}}\left(L_{1}^{\prime *}\right)$ into $\mathbf{Z} \times p^{n} \mathbf{Z}$. Since $v_{1}\left(x_{2}\right)=(0,1), x_{2}^{\prime}$ is not a norm from $L_{1}^{\prime}$ for $l<p^{n}$; hence the cyclic algebra $A_{1} \otimes_{F} L_{1}$ has exponent at least $p^{n}$ (cf. [R, p. 261, Corollary 30.7]). The exponent divides the index, so equals $p^{n}$. Turning to $L_{2}$, we have $x_{1} \in L_{2}^{* p^{n}}$ by Lemma 1.9(ii) since $x_{1}$ is a unit of $\left(L_{2}, v_{2}\right)$ which is strictly $p$-Henselian; hence $L_{2}$ splits $A_{1}$.

(ii) The arguments of (i) for $A_{1}$ apply to $A_{2}$ with the valuations reversed, yielding the first part of (ii). Now, $A_{2} \otimes_{F} L_{2}$ corresponds to $\left(y_{1}\right) \cup\left(y_{2}\right)+\cdots+\left(y_{2 m-1}\right) \cup$ $\left(y_{2 m}\right)$ in $H_{p}^{2}\left(L_{2}, \mu_{p}\right) \cong H^{2}\left(P_{2 m}, \mathbf{Z} / p \mathbf{Z}\right)$. Thus, Theorem 3.4 establishes the final assertion of (ii).

THEOREM 5.4. Let $D$ be the F-central division algebra defined at the beginning of this section. Then $D$ has exponent $p^{n}$ and index $p^{m}$ where $m \geqslant n \geqslant 3$. Further,

(i) $D$ is not a crossed product.

(ii) The matrix algebra $M_{p^{\prime}}(D)$ is not a crossed product for all integers $r \leqslant n-3$.

(iii) $M_{p^{n-2}}(D)$ is a crossed product but is not isomorphic to a tensor product of cyclic algebras.

(iv) $M_{p^{n-1}}(D)$ is isomorphic to the tensor product of a cyclic algebra of index $p^{n}$ and $(m-1)$ cyclic algebras of index $p$.

(v) D has a maximal subfield whose normal closure over $F$ is of degree a power of $p$.

Proof. Recall that $D$ is the underlying division algebra of $A=A_{1} \otimes_{F} A_{2}$. So, in $\operatorname{Br}\left(L_{i}\right),\left[D \otimes_{F} L_{i}\right]=\left[A \otimes_{F} L_{i}\right]=\left[A_{i} \otimes_{F} L_{i}\right], i=1,2$, by Lemma 5.3. We have $\exp (D) \leqslant p^{n}$ by the construction of the $A_{i}$, and $\exp (D) \geqslant \exp \left(A_{1} \otimes_{F} L_{1}\right)=p^{n}$, by Lemma 5.3. Applying 5.3 and the local global principles Theorem 4.11(iii), (ii), we have index $\left(D \otimes_{F} L_{i}\right)=p$-ind $\left(D \otimes_{F} L_{i}\right), i=1,2$, hence index $(D)=p$-ind $(D)=$ $\max \left\{p\right.$-ind $\left.\left(D \otimes_{F} L_{i}\right) \mid i=1,2\right\}=p^{m}$. Part (v) is a restatement of the equality index $(D)=p$-ind $(D)$.

Part (i) is a special case of (ii), so we prove (ii). Suppose $M_{p^{r}}(D)$ is a crossed product for $0 \leqslant r \leqslant n-3$. This means that there is a splitting field $K$ of $D$ with $K$ Galois over $F$ and $[K: F]=p^{m+r}$. Then $K \cdot L_{i}$ is Galois over $L_{i}$ and $\left[K \cdot L_{i}: L_{i}\right] \mid[K: F]$, so $K \cdot L_{i} \subseteq\left(\tilde{L}_{i}\right)_{p}$. Let $G_{i}=\mathscr{G}\left(K \cdot L_{i} / L_{i}\right)$. Then $G_{1}$ is a homomorphic image of $G_{p}\left(L_{1}\right) \cong\left(\hat{\mathbf{Z}}_{p}\right)^{2}$, so $G_{1}$ is abelian of rank $(:=$ minimum number of generators) $\leqslant 2$. Hence, the $p$-torsion group ${ }_{p} G_{1}$ of $G_{1}$ has order equal to $\left|G_{1} / G_{1}^{p}\right| \leqslant p^{2}$. Also, since $K \cdot L_{1}$ splits $D$ and hence splits $A_{1} \otimes_{F} L_{1}$, we have $\left|G_{1}\right|=\left[K \cdot L_{1}: L_{1}\right] \geqslant \operatorname{index}\left(A_{1} \otimes_{F} L_{1}\right)=p^{n}$ (cf. (1.1)). On the other hand, as $K \cdot L_{2}$ 
splits $A_{2} \otimes_{F} L_{2}$, the last part of Lemma 5.3 says $G_{2}$ has $(\mathbf{Z} / p \mathbf{Z})^{m}$ as a homomorphic image. Therefore, the abelian group $G_{2}$ has a subgroup $G_{3}$ with $G_{3} \cong(\mathbf{Z} / p \mathbf{Z})^{m}$. Both $G_{1}$ and $G_{3}$ may be viewed as subgroups of $\mathscr{G}(K / F)$. Since $|\mathscr{G}(K / F)|=p^{m+r}$ $\leqslant p^{m+n-3}$ while $\left|G_{1}\right| \geqslant p^{n}$ and $\left|G_{3}\right|=p^{m}$, we find $\left|G_{1} \cap G_{3}\right| \geqslant p^{3}>\left.\right|_{p} G_{1} \mid$. But $G_{1} \cap G_{3} \subseteq_{p} G_{1}$. This contradiction proves (ii).

(iii) $A_{1} \otimes_{F} L_{1}$ has a splitting field $M_{1}=L_{1}\left(x_{1}^{1 / p^{n-1}}, x_{2}^{1 / p}\right)$ which is Galois over $L_{1}$ with $\mathscr{G}\left(M_{1} / L_{1}\right) \cong \mathbf{Z} / p^{n-1} \mathbf{Z} \oplus \mathbf{Z} / p \mathbf{Z}$. Likewise $A_{2} \otimes_{F} L_{2}$ has a splitting field $M_{2}$ $=L_{2}\left(y_{1}^{1 / p}, y_{3}^{1 / p}, \ldots, y_{2 m-1}^{1 / p}\right)$ which is Galois over $L_{2}$ with $\mathscr{G}\left(M_{2} / L_{2}\right) \cong(\mathbf{Z} / p \mathbf{Z})^{m}$. Since each $M_{i}$ splits $D$, the local global principle (Theorem 4.11) (v) says that there is a splitting field $M$ of $D$ with $M$ Galois over $F$ and $\mathscr{G}(M / F) \cong \mathbf{Z} / p^{n-1} \mathbf{Z} \oplus$ $(\mathbf{Z} / p \mathbf{Z})^{m-1}$. Thus $M$ is a maximal subfield of $M_{p^{n-2}}(D)$, which must therefore be a crossed product.

Suppose $M_{p^{n-2}}(D) \cong C_{1} \otimes_{F} C_{2} \otimes_{F} \cdots \otimes_{F} C_{t}$, with each $C_{i}$ a cyclic algebra. Let $N$ be a compositum of $t$ maximal subfields cyclic over $F$, one from each $C_{i}$. Then $\mathscr{G}(N / F)$ is an abelian $p$-group, and $t \geqslant \operatorname{rank}(\mathscr{G}(N / F)) \geqslant \operatorname{rank} \mathscr{G}\left(N \cdot L_{2} / L_{2}\right) \geqslant m$; the last inequality comes from Lemma 5.3 as $N \cdot L_{2}$ splits $A_{2} \otimes_{F} L_{2}$. However, at least one of the $C_{i}$ has exponent (hence index) at least $\exp (D)=p^{n}$. Thus, $\operatorname{dim}_{F}\left(C_{1} \otimes_{F} \cdots \otimes_{F} C_{t}\right) \geqslant\left(p^{n} \cdot p^{m-1}\right)^{2}>\operatorname{dim}_{F} M_{p^{n-2}}(D)$, and this contradiction finishes (iii).

For (iv) note that $A_{\omega}\left(x_{1}, y_{2}^{p^{n-1}} ; F\right)$ and $A_{\omega}\left(y_{1}, x_{2} ; F\right)$ are split, by Theorem 5.1(iii), since the argument of Lemma 5.3 shows that they are each split by $L_{1}$ and by $L_{2}$. Also, in $\operatorname{Br}_{p}(F),\left[A_{\omega}\left(y_{1}, y_{2}^{p^{n-1}} ; F\right)\right]=\left[A_{\rho}\left(y_{1}, y_{2} ; F\right)\right]$ by $[\mathbf{R}$, p. 262, Theorem 30.10] as $\rho=\omega^{p^{n}-1}$. Thus, in $\operatorname{Br}_{p}(F), D$ is similar to $A_{1} \otimes_{F} A_{2}$ which is similar to

$$
A_{\omega}\left(x_{1} y_{1}, x_{2} y_{2}^{p^{n-1}} ; F\right) \otimes_{F} A_{\rho}\left(y_{3}, y_{4} ; F\right) \otimes_{F} \cdots \otimes_{F} A_{\rho}\left(y_{2 m-1}, y_{2 m} ; F\right) \text {. }
$$

This yields (iv), completing the proof of the theorem.

REMARKS 5.5. (i) In case $m=n$ we can see that $D$ is not a crossed product using the valuation theory in $\$ 2$ without invoking the cohomological machinery in $\$ 3$. (Indeed, $\$ 3$ is needed only for working with matrix algebras in proving Theorem 5.4(ii), (iii).) For, suppose $K$ is a maximal subfield of $D$ with $K$ Galois over $F$. Let $G=\mathscr{G}(K / F)$. Because $D \otimes_{F} L_{i} \cong A_{i} \otimes_{F} L_{i}$ is a division algebra by Lemma 5.3 and Example 2.7, $K$ is linearly disjoint to $L_{i}$ over $F$, so that $G \cong \mathscr{G}\left(K \cdot L_{i} / L_{i}\right)$. By 2.7, $\mathscr{G}\left(K \cdot L_{i} / L_{i}\right)$ is isomorphic to a subgroup of $\Gamma_{A_{i} \otimes_{F} L_{i}} / \Gamma_{L_{i}}$; but this group is $\left(\mathbf{Z} / p^{m} \mathbf{Z}\right)^{2}$ if $i=1$ and $(\mathbf{Z} / p \mathbf{Z})^{2 m}$ if $i=2$. Clearly these two groups have no common subgroup of order $p^{m}$, as $m \geqslant 3$, so $D$ cannot be a crossed product.

(ii) An explicit example (for any $m \geqslant n \geqslant 3$ ) of a maximal subfield of $D$ is given by $K=F\left(\alpha_{1}, \alpha_{2}, \ldots, \alpha_{m}\right)$, where

$$
\begin{aligned}
& \alpha_{1}=\sqrt[p]{\left(x_{1}+y_{2 m}\right)^{p-1} x_{1} y_{2 m}} \\
& \alpha_{j}=\sqrt[p]{\left(\alpha_{j-1}+y_{2(m-j+1)}\right)^{p-1} \alpha_{j-1} y_{2(m-j+1)}}, \quad j=2,3, \ldots, m .
\end{aligned}
$$

One can check that $K \cdot L_{1}=L_{1}\left(x_{1}^{1 / p^{m}}\right)$ and $K \cdot L_{2}=L_{2}\left(y_{2}^{1 / p}, y_{4}^{1 / p}, \ldots, y_{2 m}^{1 / p}\right)$. Since $K \cdot L_{i}\left(\cong K \otimes_{F} L_{i}\right)$ splits $A_{i}$, hence $D$, Theorem 4.11(iv) shows $K$ splits $D$. This gives a more concrete verification of the index of $D$ and of (v) of the theorem. 
(iii) We have focussed here on a single prime $p$. But if we take the $L_{i}$ to be strict Henselizations of $\left(F_{0}, v_{i}\right)$ (not just strict $p$-Henselizations) and assume $k$ has enough roots of unity, it is clear that we can find over $F=L_{1} \cap L_{2}$ noncrossed products $D_{q}$ of index $q^{m}$ and exponent $q^{n}(m \geqslant n \geqslant 3)$ for every prime $q \neq$ char $k$. It can be shown that $M_{t}\left(D_{q}\right)$ is a crossed product iff $q^{n-2} \mid t$. Furthermore, noncrossed products of composite index can be constructed over such an $F$.

REMARK 5.6. When $m>n$ the noncrossed product $D$ of Theorem 5.4 is decomposable-one can check that $D \cong D_{0} \otimes_{F} D_{1} \otimes_{F} \cdots \otimes_{F} D_{m-n}$, where $D_{0}$ is a noncrossed product of exponent and index $p^{n}$, while $D_{1}, \ldots, D_{m-n}$ are cyclic of exponent and index $p$. However, the methods used in constructing $D$ can also be applied to obtain examples of indecomposable division algebras with index exceeding the exponent. Here is a sketch for the case index $=p^{4}$, exponent $=p^{3}$ (which is inspired by the examples in [ $\left.\left.\mathbf{S a}_{\mathbf{3}}, \S 2\right]\right)$ : Construct fields and valuations $\left(L_{1}, v_{1}\right)$, $\left(L_{2}, v_{2}\right)$ and $F=L_{1} \cap L_{2}$ exactly as at the beginning of this section except with four $x_{i}$ instead of two and four $y_{i}$. Let $\omega_{j}$ be a primitive $p^{j}$ th root of unity in $F$, $j=1,2,3$, let

$$
\begin{aligned}
& A_{1}=A_{\omega_{3}}\left(x_{1}, x_{2} ; F\right) \otimes_{F} A_{\omega_{1}}\left(x_{3}, x_{4} ; F\right), \\
& A_{2}=A_{\omega_{2}}\left(y_{1}, y_{2} ; F\right) \otimes_{F} A_{\omega_{2}}\left(y_{3}, y_{4} ; F\right),
\end{aligned}
$$

and let $D$ be the underlying division algebra of $A_{1} \otimes_{F} A_{2}$. One checks as in Lemma 5.3 and Theorem 5.4 that $\operatorname{index}(D)=p^{4}, \exp (D)=p^{3}$, and that $D_{i}:=D \otimes_{F} L_{i} \cong$ $A_{i} \otimes_{F} L_{i}, i=1,2$. Furthermore, by Corollary 2.6 the valuation $v_{i}$ on $L_{i}$ extends to $D_{i}$, so $D_{i}$ is a division algebra totally ramified over $L_{i}$, and $\Gamma_{D_{1}} / \Gamma_{L_{1}} \cong\left(\mathbf{Z} / p^{3} \mathbf{Z}\right)^{2} \times$ $(\mathbf{Z} / p \mathbf{Z})^{2}$ and $\Gamma_{D_{2}} / \Gamma_{L_{2}} \cong\left(\mathbf{Z} / p^{2} \mathbf{Z}\right)^{4}$. Suppose $D_{i}=D_{\alpha} \otimes_{L_{i}} D_{\beta}$. We claim that $\Gamma_{D_{\alpha}} \cap$ $\Gamma_{D_{\beta}}=\Gamma_{L_{i}}$. For, otherwise $\left(\Gamma_{D_{\alpha}} \cap \Gamma_{D_{\beta}}\right) / \Gamma_{L_{i}}$ would have a nontrivial cyclic subgroup $H$, and $D_{\alpha}$ and $D_{\beta}$ would each contain a copy of the unique totally ramified field extension $K$ of $L_{i}$ with $\Gamma_{K} / \Gamma_{L_{i}}=H$. But then $D_{\alpha} \otimes_{F} D_{\beta}$ would have zero divisors, contradicting the fact that $D_{i}$ is a division algebra. This shows that $\Gamma_{D_{i}} / \Gamma_{L_{i}}=$ $\left(\Gamma_{D_{\alpha}} / \Gamma_{L_{i}}\right) \times\left(\Gamma_{D_{\beta}} / \Gamma_{L_{i}}\right)$. Note also that the invariant factors of the finite abelian groups $\Gamma_{D_{\gamma}} / \Gamma_{L_{i}}$ occur with even multiplicity, $\gamma=\alpha$, $\beta$. (For this "local" information, proofs will appear in $\left[\mathbf{W}_{2}\right]$.) Thus, in a nontrivial decomposition of $D_{1}$ one of the tensor factors has index $p^{3}$ and the other has index $p$; likewise in a decomposition of $D_{2}$ each factor has index $p^{2}$. Since the decompositions of $D_{1}$ and $D_{2}$ are incompatible, $D$ must be indecomposable. This $D$ is a crossed product, since by Theorem 4.11(v) it is split by a Galois extension $M$ of $F$ with $\mathscr{G}(M / F) \cong\left(\mathrm{Z} / p^{2} \mathbf{Z}\right)$ $\times(\mathbf{Z} / p \mathbf{Z})^{2}$. However, with suitable modifications in the construction, by using three valuations, one can obtain examples of noncrossed product division algebras which are indecomposable of degree $p^{m}$ and exponent $p^{n}$ for any of the $p^{m}$ and $p^{n}$ given in Saltman's theorem [Sa $\mathbf{S}$, p. 811, Theorem 2.6].

6. Noncrossed products of exponent $p^{2}$. We will now show that our basic method can be used to construct noncrossed product division algebras of exponent $p^{2}$ $(p \neq 2)$ and index $p^{m}$ for any $m \geqslant 2$. The construction is more delicate than the one in $\$ 5$, as we must work with a field $F$ not containing $p$ th roots of unity, and must take care to control what happens when $\mu_{p}$ is adjoined to $F$. (We need to 
assure that the local global principles of Theorem 4.11 apply to $F\left(\mu_{p}\right)$, even though they do not apply directly to $F$ itself.) It is still an open question whether there exists a noncrossed product division algebra of index $p^{2}$ over a field containing $\mu_{p}$.

We now fix a prime $p \neq 2$ and an integer $m \geqslant 2$. Fix also a field $k$, char $k \neq p$, satisfying

(i) $\left[k\left(\mu_{p}\right): k\right]=2$;

(ii) $k$ has $m+1$ linearly disjoint cyclic Galois extensions

$$
\begin{aligned}
& \mathscr{L}, \mathscr{L}_{1}, \mathscr{L}_{2}, \ldots, \mathscr{L}_{m} \text {, with }[\mathscr{L}: k]=p^{2} \text { and }\left[\mathscr{L}_{j}: k\right]=p, \\
& j=1,2, \ldots, m .
\end{aligned}
$$

For example, one could set $k_{1}=\mathbf{R}\left(w_{1}, \ldots, w_{p^{2}}, z_{i j}, 1 \leqslant i \leqslant p, 1 \leqslant j \leqslant p\right)$ where all the $w_{i}$ and $z_{i j}$ are algebraically independent over the real numbers $\mathbf{R}$; then let $k$ be the fixed field of the group $\mathbf{Z} / p^{2} \mathbf{Z} \times(\mathbf{Z} / p \mathbf{Z})^{m}$ acting on $k_{1}$ by permuting the indeterminates, cf. [Ri, §2]. Fields $k$ satisfying (6.1) exist in characteristic 0 and in those prime characteristics $q \neq p$ such that the order of the residue of $q$ in the multiplicative group of the $\operatorname{ring} \mathbf{Z} / p \mathbf{Z}$ is even.

Let $F_{0}=k\left(x_{1}, \ldots, x_{m}, y_{1}, \ldots, y_{m}\right)$, where $x_{1}, \ldots, x_{m}, y_{1}, \ldots, y_{m}$ are algebraically independent over $k$. Let $v_{1}$ be the valuation on $F_{0}$ as described in Example 2.7, viewing $F_{0}=K\left(x_{1}, \ldots, x_{m}\right)$, where $K=k\left(y_{1}, \ldots, y_{m}\right)$. So, the value group of $\left(F_{0}, v_{1}\right)$ is $(\mathbf{Z})^{m}$ ordered lexicographically and $v_{1}\left(x_{i}\right)=(0, \ldots, 0,1,0, \ldots, 0)$ (the 1 in the $i$ th position) while $v_{1}\left(y_{i}\right)=0$, all $i$. The residue field $\overline{F_{0 v_{1}}}$ is $k\left(\bar{y}_{1}, \ldots, \bar{y}_{m}\right)$ (where $\bar{y}_{i}$ is the image of $\left.y_{i}\right)$, which is isomorphic to $k\left(y_{1}, \ldots, y_{m}\right)$. Let $v_{2}$ be the same type of valuation on $F_{0}$ but with the $x_{i}$ and $y_{i}$ interchanged. So, $\Gamma_{F_{0}, v_{2}}=(\mathbf{Z})^{m}$, $v_{2}\left(y_{i}\right)=(0, \ldots, 0,1,0, \ldots, 0)$ (the 1 in the $i$ th position) while $v_{2}\left(x_{i}\right)=0$, and $\overline{F_{0 v_{2}}}=k\left(\bar{x}_{1}, \ldots, \bar{x}_{m}\right) \cong k\left(x_{1}, \ldots, x_{m}\right)$. It is easy to see that $v_{1}$ and $v_{2}$ are independent valuations. Next set

$$
F_{1}=F_{0}\left(\sqrt[p^{2}]{x_{1}+y_{1}}, \sqrt[p]{x_{2}+y_{2}}, \ldots, \sqrt[p]{x_{m}+y_{m}}\right) .
$$

Any extension of $v_{1}$ to $F_{1}$ has residue field containing $k\left(\bar{y}_{1}^{1 / p^{2}}, \bar{y}_{2}^{1 / p}, \ldots, \bar{y}_{m}^{1 / p}\right)$, an extension of $\overline{F_{0 v_{1}}}$ of degree $p^{m+1}$. From the fundamental inequality $\sum e_{i} f_{i} \leqslant$ $\left[F_{1}: F_{0}\right]=p^{m+1}$ we see that $v_{1}$ has a unique extension (also called $v_{1}$ ) to $F_{1}$ which is inertial, hence unramified, with residue field $k\left(\bar{y}_{1}^{1 / p^{2}}, \bar{y}_{2}^{1 / p}, \ldots, \bar{y}_{m}^{1 / p}\right)$. Likewise $v_{2}$ has a unique inertial extension to $F_{1}$, with residue field $F_{0}\left(\bar{x}_{1}^{1 / p^{2}}, \bar{x}_{2}^{1 / p}, \ldots, \bar{x}_{m}^{1 / p}\right)$.

Now, let $F$ be an algebraic extension of $F_{1}$ which is maximal with respect to the property that both valuations $v_{1}$ and $v_{2}$ have immediate extensions from $F_{1}$ to $F$; these valuations on $F$ are again denoted $v_{1}$ and $v_{2}$. The existence of such an $F$ follows by Zorn's lemma. This $F$ is the field over which our example will be constructed. Within the $p$ th root closure $\tilde{F}_{p}$ of $F$ let $\left(L_{i}, v_{i}\right)$ be a $p$ th root Henselization of $\left(F, v_{i}\right), i=1,2$, as described in $\S 1$. Since $F \subseteq L_{1} \cap L_{2}$ and each $v_{i}$ has an immediate extension to $L_{1} \cap L_{2}$, the definition of $F$ guarantees that $F=L_{1} \cap L_{2}$.

Let $F^{\prime}=F\left(\mu_{p}\right)$ and $L_{i}^{\prime}=L_{i}\left(\mu_{p}\right), i=1,2$. Note that $\left[F^{\prime}: F\right]=\left[L_{i}^{\prime}: L_{i}\right]=2$ by (6.1)(i) since the residue fields of the $L_{i}$ are purely transcendental over $k$. Furthermore $v_{i}$ has a unique inertial extension from $L_{i}$ to $L_{i}^{\prime}$ and from $F$ to $F^{\prime}, i=1,2$. 
The goal of the next few lemmas is to prove that $F^{\prime}=L_{1}^{\prime} \cap L_{2}^{\prime}$, so that the machinery of $\$ 4$ can be invoked. The notation defined thus far will be held fixed throughout this section.

Lemma 6.2. $F \cap L_{1}^{p} \cap L_{2}^{p}=F^{p}$.

Proof. Take any $a \in F \cap L_{1}^{p} \cap L_{2}^{p}$, and suppose $a \notin F^{p}$. Pick $\alpha_{i} \in L_{i}$ with $\alpha_{i}^{p}=a, i=1,2$. The $p$ th root Henselization $\left(L_{i}, v_{i}\right)$ is an immediate extension of $\left(F, v_{i}\right)$, so $\left.v_{i}\right|_{F\left(\alpha_{i}\right)}$ is an immediate extension of $\left(F, v_{i}\right)$ to $F\left(\alpha_{i}\right)$. Now, the polynomial $X^{p}-a \in F[X]$ is irreducible since it has no roots in $F$ and $p$ is prime (cf. [K, p. 62]). Therefore, $F\left(\alpha_{1}\right) \cong F\left(\alpha_{2}\right)$. Hence both $v_{1}$ and $v_{2}$ have immediate extensions to $F\left(\alpha_{1}\right)$, contradicting the maximality of $F$. Thus, we must have $a \in F^{p}$.

LemMa 6.3. Pick any $\tau_{i} \in G_{p}\left(L_{i}\right)=\mathscr{G}\left(\tilde{F}_{p} / L_{i}\right)$ such that $\tau_{i}$ restricts to the nontrivial $L_{i}$-automorphism of $L_{i}^{\prime}=L_{i}\left(\mu_{p}\right)$. Take any $b \in F^{\prime} \cap L_{1}^{\prime p} \cap L_{2}^{\prime p}$ and any $\beta \in L_{1}^{\prime} \cap L_{2}^{\prime}$ with $\beta^{p}=b$. Then $\tau_{1}(\beta)=\tau_{2}(\beta)$.

Proof. We have $\beta \tau_{i}(\beta)=N_{L_{i}^{\prime} / L_{i}}(\beta) \in L_{i}, i=1$, 2. Thus, $\left(\beta \tau_{i}(\beta)\right)^{p}=b \tau_{i}(b)=$ $N_{F^{\prime} / F}(b) \in F \cap L_{1}^{p} \cap L_{2}^{p}$. The preceding lemma says there is a $c \in F$ with $c^{p}=$ $\left(\beta \tau_{i}(\beta)\right)^{p}$; then $\beta \tau_{i}(\beta)=\omega_{i} c$ for some $\omega_{i} \in \mu_{p}, i=1$, 2. So $\omega_{i}=\beta \tau_{i}(\beta) c^{-1} \in L_{i}$. Since $L_{i}\left(\mu_{p}\right) \neq L_{i}$ we must have $\omega_{1}=\omega_{2}=1$. Therefore, $\tau_{1}(\beta)=c \beta^{-1}=\tau_{2}(\beta)$, as desired.

Lemma 6.4. Let $G$ be a profinite group which is generated topologically by closed subgroups $G_{1}$ and $G_{2}$. Let $H$ be an open subgroup of $G$ with $|G: H|=$ $\left|G_{1}: G_{1} \cap H\right|=\left|G_{2}: G_{2} \cap H\right|=2$. If $\tau_{i} \in G_{i}-H$, then $H$ is generated topologically by its closed subgroups $G_{1} \cap H, G_{2} \cap H$, and $\overline{\left\langle\tau_{1} \tau_{2}^{-1}\right\rangle}$.

Proof. Let $H_{0}$ be the closed subgroup of $H$ generated topologically by $G_{1} \cap H$ and $G_{2} \cap H$ and by $\overline{\left\langle\tau_{1} \tau_{2}^{-1}\right\rangle}$ (which is the closed subgroup of $H$ generated by $\tau_{1} \tau_{2}^{-1}$ ). We must show that $H_{0}=H$. Assume first that $G$ is finite. Then, as $G$ is generated by $G_{1}$ and $G_{2}$ we may express any $h \in H$ as $h=r_{1} s_{1} r_{2} s_{2} \cdots r_{n} s_{n}$, with $r_{1}, \ldots, r_{n} \in$ $G_{1}$ and $s_{1}, \ldots, s_{n} \in G_{2}$. We show by induction on $n$ that $h \in H_{0}$. For $n=1$, if $r_{1} \in H$, then also $s_{1} \in H$, so $r_{1} s_{1} \in H_{0}$. If $r_{1} \notin H$, then $s_{1} \notin H$, so that $r_{1} \tau_{1}^{-1} \in G_{1}$ $\cap H, \tau_{2} s_{1} \in G_{2} \cap H$, and $h=\left(r_{1} \tau_{1}^{-1}\right)\left(\tau_{1} \tau_{2}^{-1}\right)\left(\tau_{2} s_{1}\right) \in H_{0}$. Now assume $n>1$. If $r_{1} s_{1} \in H$, then $r_{1} s_{1} \in H_{0}$ and $r_{2} s_{2} \cdots r_{n} s_{n} \in H_{0}$ by induction, and we are done. If $r_{1} s_{1} \notin H$, then $r_{1}\left(s_{1} \tau_{2}^{-1}\right) \in H$ and $\left(\tau_{1} r_{2}\right) s_{2} r_{3} s_{3} \cdots r_{n} s_{n} \in H$. By induction both these terms lie in $H_{0}$, whence $h=\left(r_{1} s_{1} \tau_{2}^{-1}\right)\left(\tau_{2} \tau_{1}^{-1}\right)\left(\tau_{1} r_{2} s_{2} \cdots r_{n} s_{n}\right) \in H_{0}$. This proves the lemma if $G$ is finite.

Now drop the assumption that $G$ is finite. If $H_{0} \neq H$, then $H-H_{0}$ is a nonempty open subset of $H$. Since a base of open sets of $H$ is given by cosets of open normal subgroups of $H$, there is an $h \in H$ and an open normal subgroup $U$ of $H$ with $h U \cap H_{0}=\varnothing$. We may assume that $U$ is actually normal in $G$ (replacing $U$ if necessary by the finite intersection of conjugates of $U$ ). Because $U$ is open, $|G: U|<\infty$. Let $\pi: G \rightarrow G / U$ be the canonical projection. It is easy to check that the hypotheses relating $G, G_{1}, G_{2}, H, \tau_{1}, \tau_{2}$ all carry over to $\pi(G), \pi\left(G_{1}\right), \pi\left(G_{2}\right)$, $\pi(H), \pi\left(\tau_{1}\right), \pi\left(\tau_{2}\right)$. Clearly $\pi\left(H_{0}\right)$ contains the subgroup of $\pi(H)$ generated by 
$\pi(H) \cap \pi\left(G_{1}\right), \quad \pi(H) \cap \pi\left(G_{2}\right)$, and $\left\langle\pi\left(\tau_{1}\right) \pi\left(\tau_{2}\right)^{-1}\right\rangle$. But $\pi(h) \in \pi(H)-\pi\left(H_{0}\right)$ which contradicts the finite case of the lemma proved above. Thus, $H_{0}=H$, and the lemma is proved in general.

Lemma 6.5. $F^{\prime} \cap L_{1}^{\prime p} \cap L_{2}^{\prime p}=F^{\prime p}$. Consequently, $F^{\prime}=L_{1}^{\prime} \cap L_{2}^{\prime}, \quad G_{p}\left(F^{\prime}\right)=$ $G_{p}\left(L_{1}^{\prime}\right){ }_{p} G_{p}\left(L_{2}^{\prime}\right)$, and the local global principles of Theorem 4.11 hold from $L_{1}^{\prime}$ and $L_{2}^{\prime}$ to $F^{\prime}$.

Proof. We have $F \subseteq L_{i} \subseteq \tilde{F}_{p}, i=1,2$, and $F=L_{1} \cap L_{2}$. So, $G_{p}(F)=\mathscr{G}\left(\tilde{F}_{p} / F\right)$ is generated topologically by its closed subgroups $G_{p}\left(L_{1}\right)$ and $G_{p}\left(L_{2}\right)$. We have $\left|G_{p}(F): G_{p}\left(F^{\prime}\right)\right|=\left[F^{\prime}: F\right]=2$ and $G_{p}\left(F^{\prime}\right) \cap G_{p}\left(L_{i}\right)=G_{p}\left(L_{i}^{\prime}\right)$, which has index 2 in $G_{p}\left(L_{i}\right)$. Thus, Lemma 6.4 says $G_{p}\left(F^{\prime}\right)$ is generated topologically by $G_{p}\left(L_{1}^{\prime}\right), G_{p}\left(L_{2}^{\prime}\right)$, and $\overline{\left\langle\tau_{1} \tau_{2}^{-1}\right\rangle}$ for any $\tau_{i} \in G_{p}\left(L_{i}\right)$ which restricts to the nontrivial $L_{i}$-automorphism of $L_{i}^{\prime}$.

Now pick any $b \in F^{\prime} \cap L_{1}^{\prime p} \cap L_{2}^{\prime p}$, and any $\beta \in \tilde{F}_{p}$ with $\beta^{p}=b$. Let $N=$ $G_{p}\left(F^{\prime}(\beta)\right)$, a closed subgroup of $G_{p}\left(F^{\prime}\right)$. Since $L_{i}^{\prime}$ contains one, hence all $p$ th roots of $b, \beta \in L_{i}^{\prime}$, so $G_{p}\left(L_{i}^{\prime}\right) \subseteq N, i=1$, 2. But Lemma 6.3 shows $\tau_{1} \tau_{2}^{-1} \in N$ also. Since a topological generating set of $G_{p}\left(F^{\prime}\right)$ lies in $N, N=G_{p}\left(F^{\prime}\right)$ which shows that $\beta \in F^{\prime}$. Thus $F^{\prime} \cap L_{1}^{\prime p} \cap L_{2}^{\prime p}=F^{\prime p}$.

Since $F^{\prime} \subseteq L_{1}^{\prime} \cap L_{2}^{\prime} \subseteq \tilde{F}_{p}, L_{1}^{\prime} \cap L_{2}^{\prime}$ is obtainable from $F^{\prime}$ by successive adjunctions of $p$ th roots (cf. (1.4)). Thus, the equality proved in the previous paragraph implies $F^{\prime}=L_{1}^{\prime} \cap L_{2}^{\prime}$. Because $L_{i}$ is a $p$ th root Henselization of $\left(F, v_{i}\right)$, the unique extension of $v_{i}$ to $L_{i}^{\prime}$ is $p$-Henselian and is an immediate extension of $\left(F^{\prime}, v_{i}\right)$. Also, $v_{1}$ and $v_{2}$ are independent valuations on $F^{\prime}$ since they are independent on $F_{0}$ and $F^{\prime}$ is algebraic over $F_{0}$. Therefore, Theorem 4.3 shows that $G_{p}\left(F^{\prime}\right)=G_{p}\left(L_{1}^{\prime}\right){ }_{p} G_{p}\left(L_{2}^{\prime}\right)$, completing the proof.

For each $i$ the residue field $\overline{F_{v}}$ of $F$ with respect to $v_{i}$ is the same as that of $F_{1}$; so $\overline{F_{v}}$ is a purely transcendental extension of our original ground field $k$. Hence, for the fields $\mathscr{L}, \mathscr{L}_{1}, \mathscr{L}_{2}, \ldots, \mathscr{L}_{m}$ posited in (6.1)(ii), the valuation $v_{i}$ on $F$ has a unique inertial extension to $\mathscr{L} \cdot F$ (resp. to each $\mathscr{L}_{j} \cdot F$ ) with residue field $\mathscr{L} \cdot \overline{F_{v}}$ (resp $\left.\mathscr{L}_{j} \cdot \overline{F_{v}}\right)$. So, $\mathscr{L} \cdot F, \mathscr{L}_{1} \cdot F, \ldots, \mathscr{L}_{m} \cdot F$ are linearly disjoint cyclic Galois extensions of $F$. We fix a generator $\sigma$ of $\mathscr{G}(\mathscr{L} \cdot F / F) \cong \mathbf{Z} / p^{2} \mathbf{Z}$, and generators $\sigma_{j}$ of $\mathscr{G}\left(\mathscr{L}_{j} \cdot F / F\right) \cong \mathbf{Z} / p \mathbf{Z}, j=1,2, \ldots, m$. Using the cyclic algebra notation described in $\S 1$ we set

$$
A_{1}:=A\left(\mathscr{L} \cdot F / F, \sigma, x_{1}\right) \quad \text { and } \quad A_{2}:=\bigotimes_{j=1}^{m} A\left(\mathscr{L}_{j} \cdot F / F, \sigma_{j}, y_{j}\right) .
$$

The underlying division algebra $D$ of $A_{1} \otimes_{F} A_{2}$ will provide the counterexample of this section. We first consider the local properties of the $A_{i}$.

LeMMA 6.6. (i) $A_{1} \otimes_{F} L_{1}$ is a division algebra of index and exponent $p^{2}$, while $L_{2}$ splits $A_{1}$.

(ii) $A_{2} \otimes_{F} L_{2}$ is a division algebra of index $p^{m}$ and exponent $p$, while $L_{1}$ splits $A_{2}$.

(iii) $A_{i} \otimes_{F} L_{i}^{\prime}$ has the same index and exponent as $A_{i} \otimes_{F} L_{i}, i=1,2$. 
Proof. (i) Since $\left(L_{1}, v_{1}\right)$ has the same residue field as $\left(F, v_{1}\right)$, the same argument as given just above shows $v_{1}$ has a unique inertial (hence unramified) extension to $\mathscr{L} \cdot L_{1} ;$ so $\mathscr{L} \cdot F$ and $L_{1}$ are linearly disjoint over $F$. Hence, $\mathscr{G}\left(\mathscr{L} \cdot L_{1} / L_{1}\right) \cong$ $\mathscr{G}(\mathscr{L} \cdot F / F) \cong \mathbf{Z} / p^{2} \mathbf{Z}$ and $A_{1} \otimes_{F} L_{1} \cong A\left(\mathscr{L} \cdot L_{1} / L_{1}, \sigma, x_{1}\right)$. Since $v_{1}\left(x_{1}\right)=$ $(1,0, \ldots, 0)$ in the value group $\Gamma_{L_{1}}$ of $L_{1}$, the image of $v_{1}\left(x_{1}\right)$ in $\Gamma_{L_{1}} / p^{2} \Gamma_{L_{1}}$ has order $p^{2}$. Therefore, Corollary 2.9 with $k=1$ and $n_{1}=l=p^{2}$ shows that $A_{1} \otimes_{F} L_{1}$ is a valued division algebra; its index is clearly $p^{2}$. Because $v_{1}$ extends uniquely to $\mathscr{L} \cdot L_{1}$ without ramification, $v_{1}$ maps the norm group $N_{\mathscr{L} \cdot L_{1} / L_{1}}\left(\mathscr{L} \cdot L_{1}^{*}\right)$ into $p^{2} \Gamma_{L_{1}}$. Thus, $x_{1}^{r}$ cannot be a norm from $\mathscr{L} \cdot L_{1}$ to $L_{1}$ for $1 \leqslant r<p^{2}$; this shows that $A_{1} \otimes_{F} L_{1}$ has exponent $p^{2}$ by [R, p. 261, Corollary 30.7].

Now consider $A_{1} \otimes_{F} L_{2}$. We have again that $\mathscr{L} \cdot F$ is linearly disjoint to $L_{2}$ over $F$, so $A_{1} \otimes_{F} L_{2} \cong A\left(\mathscr{L} \cdot L_{2} / L_{2}, \sigma, x_{1}\right)$. But $\bar{x}_{1}$ has a $p^{2}$-root in $\bar{L}_{2}$. Thus, the polynomial $f(X)=X^{p^{2}}-x_{1} \in V_{L_{2}}[X]$, which splits over $\tilde{F}_{p}$, has image $\bar{f}$ in $\bar{L}_{2}[X]$ with a nonrepeated linear factor. Because $\left(L_{2}, v_{2}\right)$ is $p$ th root Henselian, $f$ must have a linear factor in $V_{L_{2}}[X]$, i.e., $x_{1}$ has a $p^{2}$-root in $L_{2}$. Therefore, $x_{1}$ lies in the norm group $N_{\mathscr{L} \cdot L_{2} / L_{2}}\left(\mathscr{L} \cdot L_{2}^{*}\right)$, which shows that $A_{1} \otimes_{F} L_{2}$ is split.

(ii) As in (i), but with the valuations reversed, we see that $v_{2}$ has a unique inertial extension to $\mathscr{L}_{j} \cdot L_{2}$ for $j=1,2, \ldots, m$, with residue field $\mathscr{L}_{j} \cdot \bar{L}_{2}$. Hence, $A\left(\mathscr{L}_{j} \cdot F / F, \sigma_{j}, y_{j}\right) \otimes_{F} L_{2} \cong A\left(\mathscr{L}_{j} \cdot L_{2} / L_{2}, \sigma_{j}, y_{j}\right)$. Corollary 2.9 applies to the tensor product of these algebras with $k=m, n_{1}=n_{2}=\cdots=n_{k}=l=p$, showing that $A_{2} \otimes_{F} L_{2} \cong \otimes_{j=1}^{m} A\left(\mathscr{L}_{j} \cdot L_{2} / L_{2}, \sigma_{j}, y_{j}\right)$ is a valued division algebra with residue ring $\mathscr{L}_{1} \cdot \ldots \mathscr{L}_{m} \cdot \bar{L}_{2}$. Clearly the exponent of $A_{2} \otimes_{F} L_{2}$ is $p$ and the index is $p^{m}$. Switching to $L_{1}$ we find that $A_{2} \otimes_{F} L_{1} \cong \otimes_{j=1}^{m} A\left(\mathscr{L}_{j} \cdot L_{1} / L_{1}, \sigma_{j}, y_{j}\right)$. But as each $\bar{y}_{j}$ has a $p$ th root in $\bar{L}_{1}$ an argument like that in (i) shows that each $y_{j}$ has a $p$ th root in $L_{1}$. Hence, $L_{1}$ splits $A_{2}$ as it splits each of the cyclic factors.

(iii) The same arguments just given for $A_{i} \otimes_{F} L_{i}$ apply to $A_{i} \otimes_{F} L_{i}^{\prime}$. Alternatively, note that $\left[L_{i}^{\prime}: L_{i}\right]=\left[L_{i}\left(\mu_{p}\right): L_{i}\right]=2$. Hence, the index reduction formula [P, p. 243] shows that for any central simple $L_{i}$-algebra $B$ of odd index, index $\left(B \otimes_{L_{i}} L_{i}^{\prime}\right)=$ index $(B)$. So, in particular, the map $\operatorname{Br}_{p}\left(L_{i}\right) \rightarrow \mathrm{Br}_{p}\left(L_{i}^{\prime}\right)$ is injective.

Let $A=A_{1} \otimes_{F} A_{2}$ with the $A_{i}$ as defined before Lemma 6.6 and the $F$ defined at the beginning of $\S 6$. Write $A \cong M_{l}(D)$, where $D$ is an $F$-central division algebra.

THEOREM 6.7. The division algebra $D$ just defined has index $p^{m}$ and exponent $p^{2} . D$ is not a crossed product. The $F\left(\mu_{p}\right)$-division algebra $D \otimes_{F} F\left(\mu_{p}\right)$, with the same index and exponent as $D$, is a crossed product.

Proof. Observe that Lemma 6.6 shows that $\left[D \otimes_{F} L_{i}\right]=\left[A_{i} \otimes_{F} L_{i}\right]$ in $\operatorname{Br}_{p}\left(L_{i}\right)$ and $\left[D \otimes_{F} L_{i}^{\prime}\right]=\left[A_{i} \otimes_{F} L_{i}^{\prime}\right]$ in $\mathrm{Br}_{p}\left(L_{i}^{\prime}\right)$. The construction of the $A_{i}$ shows that $\exp (D) \mid p^{2}$. Then $\exp (D)=p^{2}$ since $\exp \left(D \otimes_{F} L_{1}\right)=p^{2}$ by Lemma 6.6(i). Hence, $\operatorname{index}(D)=p^{s}$ for some $s \geqslant 2$. By the index reduction formula [P, p. 243], index $(D)$ $=\operatorname{index}\left(D \otimes_{F} F^{\prime}\right)$ as $\left[F^{\prime}: F\right]=2$ is prime to $p$. We compute the index of $D \otimes_{F} F^{\prime}$ using the local global principles of Theorem 4.11. Since $A_{i} \otimes_{F} L_{i}^{\prime}$ is a crossed product division algebra, index $\left(A_{i} \otimes_{F} L_{i}^{\prime}\right)=p$-ind $\left(A_{i} \otimes_{F} L_{i}^{\prime}\right)$. Therefore, by Lemmas 6.6(iii) and 6.5, and Theorem 4.11(ii), (iii), 


$$
\begin{aligned}
p^{m} & =\max \left\{\operatorname{index}\left(A_{i} \otimes_{F} L_{i}^{\prime}\right) \mid i=1,2\right\}=\max \left\{\operatorname{index}\left(D \otimes_{F} L_{i}^{\prime}\right) \mid i=1,2\right\} \\
& =\operatorname{index}\left(D \otimes_{F} F^{\prime}\right)=\operatorname{index}(D) .
\end{aligned}
$$

By comparing indices we see that $D \otimes_{F} L_{2} \cong A_{2} \otimes_{F} L_{2}$.

Suppose $D$ is a crossed product. Then there exists a maximal subfield $K$ of $D$ with $[K: F]=p^{m}$ and $K$ Galois over $F$. Let $K_{i}=K \cdot L_{i}, i=1$, 2. Then each $K_{i}$ is Galois over $L_{i}$, and we view $\mathscr{G}\left(K_{i} / L_{i}\right) \subseteq \mathscr{G}(K / L)$ by restriction. In particular, $\left[K_{i}: L_{i}\right]$ is a power of $p$. Since $\left(L_{i}, v_{i}\right)$ is $p$-Henselian, $v_{i}$ has a unique extension to a valuation of $K_{i}$. According to Corollary $2.4, K_{i}$ is an inertial extension of $L_{i}$ with $\mathscr{G}\left(K_{i} / L_{i}\right) \cong \mathscr{G}\left(\bar{K}_{i} / \bar{L}_{i}\right), i=1,2$.

Since $D \otimes_{F} L_{2} \cong A_{2} \otimes_{F} L_{2}$ is a division algebra, $K_{2} \cong K \otimes_{F} L_{2}$, which is isomorphic to a maximal subfield $K_{3}$ of $A_{2} \otimes_{F} L_{2}$. Hence $\left[K_{3}: L_{2}\right]=p^{m}$ and $\mathscr{G}(K / F) \cong$ $\mathscr{G}\left(K_{3} / L_{2}\right) \cong \mathscr{G}\left(\bar{K}_{3} / \bar{L}_{2}\right)$. As we saw in proving Lemma 6.6(ii) $A_{2} \otimes_{F} L_{2}$ is a valued division algebra with residue ring $\mathscr{L}_{1} \cdots \cdot \mathscr{L}_{m} \cdot \bar{L}_{2}$. Thus $\bar{K}_{3} \subseteq \mathscr{L}_{1} \cdots \cdot \mathscr{L}_{m} \cdot \bar{L}_{2}$, and equality must hold by comparing degrees over $\bar{L}_{2}$. Since each $\mathscr{L}_{i}$ was a cyclic extension of $k$ of degree $p, \mathscr{G}\left(\bar{K}_{3} / \bar{L}_{2}\right) \cong(\mathbf{Z} / p \mathbf{Z})^{m}$. Putting these isomorphisms together, we have $\mathscr{G}(K / F) \cong(\mathbf{Z} / p \mathbf{Z})^{m}$. Hence, the subgroup $\mathscr{G}\left(K_{1} / L_{1}\right)$ is elementary abelian.

Recall now from the proof of Lemma 6.6 that $A_{1} \otimes_{F} L_{1} \cong A\left(\mathscr{L} \cdot L_{1} / L_{1}, \sigma, x_{1}\right)$, where $\mathscr{L} \cdot L_{1}$ is a cyclic Galois and inertial extension of $L_{1}$ with $\mathscr{G}\left(\mathscr{L} \cdot L_{1} / L_{1}\right) \cong$ $\mathbf{Z} / p^{2} \mathbf{Z}$. Because $\mathscr{G}\left(K_{1} / L_{1}\right)$ is elementary abelian, $\mathscr{L} \cdot L_{1} \nsubseteq K_{1}$. Therefore, $\mathscr{L} \cdot K_{1}$, which is a cyclic Galois extension of $K_{1}$, has degree $p$ or $p^{2}$ over $K_{1}$. By [R, p. 261, Theorem 30.8], $A_{1} \otimes_{F} K_{1}$ is similar to $A\left(\mathscr{L} \cdot K_{1} / K_{1}, \tau, x_{1}\right)$ in $\operatorname{Br}\left(K_{1}\right)$, where $\tau=\sigma$ if $\left[\mathscr{L} \cdot K_{1}: K_{1}\right]=p^{2}$ and $\tau=\sigma^{p}$ if $\left[\mathscr{L} \cdot K_{1}: K_{1}\right]=p$. In either case, $\mathscr{L} \cdot K_{1}$ is an inertial extension of $K_{1}$ by Corollary 2.4. ( $\mu_{p} \nsubseteq K_{1}$, as $\left[K_{1}: L_{1}\right]$ is a power of $p$.) Therefore, since $K_{1}$ has the same value group as $L_{1}$ and $v_{1}\left(x_{1}\right)=(1,0, \ldots, 0)$ we see from Corollary 2.9 with $k=1$ and $l=n_{1}=p^{2}$ or $p$ that $A\left(\mathscr{L} \cdot K_{1} / K_{1}, \tau, x_{1}\right)$ is a division algebra of index $p^{2}$ or $p$. So, $K_{1}$ does not split $A_{1}$. Since $\left[A_{1} \otimes_{F} L_{1}\right]=$ [ $\left.D \otimes_{F} L_{1}\right]$ in $\operatorname{Br}_{p}\left(L_{1}\right), K_{1}$ cannot split $D$. But $K_{1}$ contains the maximal subfield $K$ of $D$. This contradiction shows $D$ cannot be a crossed product.

To see that $D \otimes_{F} F^{\prime}$ is a crossed product, where $F^{\prime}=F\left(\mu_{p}\right)$, we first work locally. We have $\mathscr{L} \cdot L_{1}^{\prime}$ is cyclic Galois over $L_{1}^{\prime}$. Hence by Kummer theory there is a cyclic subextension $L_{1}^{\prime}(\sqrt[p]{l})$ of degree $p$ over $L_{1}^{\prime}$. By [R, p. 261, Theorem 30.8], $A_{1} \otimes_{F} L^{\prime}(\sqrt[p]{l})$ is similar to $A\left(\mathscr{L} \cdot L_{1}^{\prime}(\sqrt[p]{l}) / L_{1}^{\prime}(\sqrt[p]{l}), \sigma^{p}, x_{1}\right)$ in $\operatorname{Br}_{p}\left(L_{1}^{\prime}(\sqrt[p]{l})\right)$, and this algebra is split by $M_{1}:=L_{1}^{\prime}\left(\sqrt[p]{l}, \sqrt[p]{x_{1}}\right)$. Invoking Lemma $6.6(\mathrm{i})$ we see that $D$ is split by $M_{1}$. But $A_{2}$, and hence $D$, is split by $M_{2}:=L_{2}^{\prime}\left(\sqrt[p]{y_{1}}, \ldots, \sqrt[p]{y_{m}}\right)$. Each $M_{i}$ is Galois over $L_{i}^{\prime}$ and $\mathscr{G}\left(M_{1} / L_{1}^{\prime}\right) \cong(\mathbf{Z} / p \mathbf{Z})^{2}$ while $\mathscr{G}\left(M_{2} / L_{2}^{\prime}\right) \cong(\mathbf{Z} / p \mathbf{Z})^{m}$. By Lemma 6.5 and the local global principle Theorem 4.11(v) there is a field $M$ Galois over $F^{\prime}$ such that $M$ splits $D$ and $\mathscr{G}\left(M / F^{\prime}\right) \cong(\mathbf{Z} / p \mathbf{Z})^{m}$. By dimension count $M$ is a maximal subfield of $D \otimes_{F} F^{\prime}$; hence $D \otimes_{F} F^{\prime}$ is a crossed product.

REMARK 6.8. One can show that the $p$-index of $D$, as defined in $\S 4$, is $p^{m}$, the same as its index. 


\section{REFERENCES}

[Am] S. A. Amitsur, On central division algebras, Israel J. Math. 12 (1972), 408-420.

[AEJ] J. Kr. Arason, R. Elman and B. Jacob, The graded Witt ring and Galois cohomology. I, Quadratic and Hermitian Forms (C. Riehm and I. Hambleton, eds.), Canad. Math. Soc. Conf. Proc., Vol. 4, Amer. Math. Soc., Providence, R.I., 1984, pp. 17-50.

[BNW] E. Binz, J. Neukirch and G. H. Wenzel, A subgroup theorem for free products of pro-finite groups, J. Algebra 19 (1971), 104-109.

$\left[\mathbf{B o}_{1}\right]$ N. Bourbaki, Algèbre commutative, Chapter I, Modules plats, Hermann, Paris, 1961.

$\left[\mathbf{B o}_{2}\right]$ _ Algèbre commutative, Chapter VI, Valuations, Hermann, Paris, 1964.

[Br] L. Bröcker, Characterization of fans and hereditarily Pythagorean fields, Math. Z. 151 (1976), 149-163.

[CF] J. W. S. Cassels and A. Fröhlich, eds., Algebraic number theory, Academic Press, London, 1967.

[E] O. Endler, Valuation theory, Springer-Verlag, New York, 1972.

[Er] Ju. L. Ershov, Semilocal fields, Soviet Math. Dokl. 15 (1974), 424-428.

[HS] P. J. Hilton and U. Stammbach, $A$ course in homological algebra, Springer-Verlag, New York, 1971.

[J] B. Jacob, On the structure of Pythagorean fields, J. Algebra 68 (1981), 247-267.

[Ja] N. Jacobson, P.I.-algebras: An introduction, Lecture Notes in Math., vol. 441, Springer-Verlag, New York, 1975.

[K] I. Kaplansky, Fields and rings, Univ. of Chicago Press, Chicago, Ill., 1969.

[MS] A. S. Merkurjev and A. A. Suslin, K-cohomologv of Severi-Brauer varieties and the norm residue homomorphism, Math. USSR Izv. 21 (1983), 307-340.

[Mi] J. Milnor, Algebraic K-theory and quadratic forms, Invent. Math. 9 (1970), 318-344.

[N] J. Neukirch, Freie Produkte proendlichen Gruppen und ihre Kohomologie, Arch. Math. 22 (1971), 337-357.

[P] R. S. Pierce, Associative algebras, Springer-Verlag, New York, 1982.

[R] I. Reiner, Maximal orders, Academic Press, London, 1975.

[Ri] L. Risman, Cyclic algebras, complete fields, and crossed products, Israel J. Math. 28 (1977), 113-128.

[Ro] L. H. Rowen, Division algebra counterexamples of degree 8, Israel J. Math. 38 (1981), 51-57.

$\left[\mathbf{S a}_{\mathbf{1}}\right]$ D. Saltman, Noncrossed products of small exponents, Proc. Amer. Math. Soc. $\mathbf{6 8}$ (1978), 165-168.

$\left[\mathbf{S a}_{2}\right] \ldots$, Noncrossed product p-algebras and Galois p-extensions, J. Algebra 52 (1978), 302-314.

$\left[\mathbf{S a}_{3}\right]$, Indecomposable division algebras, Comm. Algebra 7 (1979), 791-817.

[SS] M. Schacher and L. Small, Noncrossed products in characteristic p, J. Algebra 24 (1973), 100-103.

[Sch] W. Scharlau, Über die Brauer-Gruppe eines Henselkorpers, Abh. Math. Sem. Univ. Hamburg 33 (1969), 243-249.

[S] O. F. G. Schilling, The theory of valuations, Math. Surveys, no. 4, Amer. Math. Soc., Providence, R. I., 1950

[Se $\left.\mathbf{S}_{1}\right]$ J.-P. Serre, Cohomologie Galoisienne, Lecture Notes in Math., vol. 5, Springer-Verlag, Berlin, 1965.

$\left[\mathbf{S e}_{2}\right]$ _ Local fields, Springer-Verlag, New York, 1979; English transl. of Corps locaux.

[Sh] S. Shatz, Profinite groups, arithmetic, and geometry, Ann. of Math. Studies, no. 67, Princeton Univ. Press, Princeton, N. J., 1974.

[T] J. Tate, Relations between $K_{2}$ and Galois cohomologv, Invent Math. 36 (1976), 257-274.

[Ti] J.-P. Tignol, Cyclic and elementary abelian subfields of Malcev-Neumann division algebras, preprint. 1985.

[TA] J.-P. Tignol and S. A. Amitsur, Totally ramified splitting fields of central simple algebras over Henselian fields, J. Algebra (to appear).

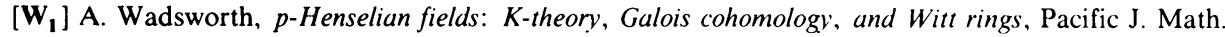
105 (1983), 473-495.

$\left[\mathbf{W}_{2}\right] \ldots$, Totally ramified valuations on finite dimensional division algebras (in preparation).

[We] E. Weiss, Cohomology of groups, Academic Press, New York, 1969.

Department of Mathematics, Oregon State University, Corvallis, Oregon 97331

Department of Mathematics, University of California, San Diego, la Jolia, California 92093 